\title{
A New Type of Compositive Information Entropy for IvIFS and Its Applications
}

\author{
Junjun Mao, ${ }^{1,2,3}$ Yuan Zhao, ${ }^{1,2}$ and Chuang $\mathrm{Ma}^{1,2}$ \\ ${ }^{1}$ School of Mathematical Sciences, Anhui University, Hefei, Anhui 230039, China \\ ${ }^{2}$ Key Laboratory of Intelligent Computing and Signal Processing of Ministry of Education, Anhui University, Hefei, \\ Anhui 230039, China \\ ${ }^{3}$ School of Mathematics, University of Manchester, Manchester M139PL, UK
}

Correspondence should be addressed to Yuan Zhao; zhaoyuan789@yahoo.com

Received 19 August 2015; Revised 30 December 2015; Accepted 4 January 2016

Academic Editor: Shuming Wang

Copyright (C) 2016 Junjun Mao et al. This is an open access article distributed under the Creative Commons Attribution License, which permits unrestricted use, distribution, and reproduction in any medium, provided the original work is properly cited.

We first show the interval-valued intuitionistic fuzzy entropy which reflects intuitionism and fuzziness of interval-valued intuitionistic fuzzy set (IvIFS) based on interval-valued intuitionistic fuzzy cross-entropy. As for intuitionism and fuzziness of IvIFS, we propose interval-valued intuitionistic entropy and interval-valued fuzzy entropy, respectively. Furthermore, we establish the interval-valued span entropy describing the uncertainty of membership degree and nonmembership degree and show some concrete measure formulas. Combining intuitionistic factor, fuzzy factor, and span factor, we ultimately put forward the axiomatic definition of the compositive entropy and give a measure formula of compositive entropy. In addition, the effectiveness of the compositive entropy measure is illuminated by comparison with other entropy measures. Furthermore, the compositive entropy is applied to multiple attributes' decision-making by using the weighted correlation coefficient between IvIFSs and pattern recognition by a similarity measure transformed from the compositive entropy.

\section{Introduction}

Since Zadeh [1] first introduced fuzzy set (FS) in 1965, many theories of higher order fuzzy set have been proposed. In 1986, Atanassov [2] generalized FS to intuitionistic fuzzy set (IFS) described by the membership degree and nonmembership degree for each element of the universe. In addition, the interval-valued fuzzy set (IvFS) [3] is conceived by Zadeh to specify the interval-valued degree of membership to each element of the universe. The concept of vague set (VS) [4] introduced by Gau and Buehrer is another generalization of fuzzy set, which is identified with IvFS pointed out in [5]. Torra [6] proposed the concept of hesitant fuzzy set (HFS) to permit the membership of an element to be a set of several possible values between 0 and 1. In 1989, Atanassov and Gargov [7] combined IFS with IvFS and introduced the notion of interval-valued intuitionistic fuzzy set (IvIFS) whose membership degree and nonmembership degree were intervals rather than real numbers. The IvIFS could also be described by a membership interval, a nonmembership interval, and a hesitancy interval, which made IvIFS more powerful and flexible in dealing with complexity and uncertainty than IvFS and IFS. In recent years, further researches about IvIFS have gained a series of achievements. Atanassov [8] presented some operations of IvIFS and studied their basic properties. Park et al. [9] investigated the correlation coefficients of IvIFS, which considered three parameters feature of IvIFS. Deschrijver and Kerre [10] established the relationships among IvIFS, IFS, and $L$-fuzzy set. In [11], Xu et al. investigated the clustering operations of IvIFS and in [12], Xu and Chen defined a variety of distance measures and similarity measures of IvIFS for decision-making.

In 1968, Zadeh [13] first introduced the entropy of fuzzy event to measure uncertain information by probabilistic methods. In the past decades, fuzzy entropy, as a very important notion for measuring fuzziness degree or uncertain 
information in fuzzy set theory, has received great attention. de Luca and Termini [14] presented the axiomatic definition of fuzzy entropy. Kaufmann [15], Yager [16], and Liu [17] defined some fuzzy entropy formulas for fuzzy set by utilizing a distance measure that describes the difference between FSs. In order to measure the uncertain information of IFS, Burillo and Bustince [18, 19] defined intuitionistic fuzzy entropy and extended this concept to interval-valued version. Szmidt and Kacprzyk [20] proposed another intuitionistic fuzzy entropy by employing a geometric interpretation of IFS. Hung and Yang [21] and Wang and Lei [22] improved entropy formula and its constructive principles, but they still ignored the effect induced by changes of hesitancy degree when membership degree is equal to nonmembership degree. So Mao et al. [23] established a novel entropy of IFS, which included two factors, the intuitionistic factor and the fuzzy factor. For IvIFS, Zhang et al. [24] and Wei et al. [25] defined the entropy of IvIFS, which generalized the entropy of IFS in [20]. Zhang et al. [26] showed the entropy measure by transforming the IvIFS into IFS. Jin et al. [27] proposed the interval-valued intuitionistic fuzzy continuous weighted entropy on the basis of the continuous ordered weighted averaging (COWA) operator. Ye [28] proposed two entropy measures for IvIFSs and established an entropy weighted model to determine the entropy weights with respect to a decision matrix provided as IvIFS. Besides, Qu et al. [29] showed a reasonable entropy formula which considered the span of membership degree and nonmembership degree.

In order to measure the discrimination information for different kinds of fuzzy sets, the cross-entropy has been widely studied. Shang and Jiang [30] defined the fuzzy cross-entropy between two FSs. Vlachos and Sergiadis [31] introduced the concept of intuitionistic fuzzy cross-entropy of IFSs and applied a kind of intuitionistic fuzzy cross-entropy measure to pattern recognition, medical diagnosis, and image segmentation. Based on intuitionism and fuzziness of IFS, Mao et al. [23] constructed a new cross-entropy to measure discrimination uncertain information between IFSs. Peng et al. [32] proposed the cross-entropy of intuitionistic hesitant fuzzy sets (IHFSs) which was developed by integrating the cross-entropy of IFSs and HFSs. By transforming IvIFS into FS, Ye [33] structured a fuzzy cross-entropy of IvIFSs.

Previous works only reflect one or two aspects of the uncertainty information of IvIFS. In fact, there are three types of uncertainty factors for IvIFS, including intuitionistic factor, fuzzy factor, and newly proposed span factor which can depict the extent of variation for the interval values of membership degree and nonmembership degree. Based on these three kinds of uncertainty factors, the main purpose of this paper is to construct a new compositive entropy which can measure uncertain information of IvIFS accurately. First, we put forward three kinds of entropy of IvIFS, including interval-valued intuitionistic entropy, interval-valued fuzzy entropy, and interval-valued span entropy. The intervalvalued span entropy is studied in particular and general measure formula for the interval-valued span entropy is presented. Then by integrating these three kinds of entropy of IvIFS, we give a measure formula of compositive entropy which meets the axiomatic definition of compositive entropy of IvIFS. Finally, we also make comparisons with other existing formulas and apply the compositive entropy measure in decision-making and pattern recognition to demonstrate its efficiency. The rest of the paper is organized as follows. In Section 2, some definitions about IFS and IvIFS are shown. In Section 3, firstly, the intuitionistic fuzzy cross-entropy and intuitionistic fuzzy entropy are presented. Then we propose another three kinds of entropy for IvIFS, including interval-valued intuitionistic entropy, interval-valued fuzzy entropy, and interval-valued span entropy. Finally, based on intuitionistic factor, fuzzy factor, and span factor, we show the axiomatic conditions of the compositive entropy and construct a measure formula. In Section 4, we compare the compositive entropy measure with other existing entropy measures. And the proposed formula is applied to multiple attributes' decision-making and pattern recognition. Conclusions are presented in Section 5.

\section{Preliminaries}

In this section, some basic concepts are illustrated, which will be needed in the following analysis.

Definition 1 (see [2]). Let a nonempty set $X$ be the universe of discourse; an intuitionistic fuzzy set on $X$ is defined by Atanassov as $A=\left\{\left\langle x, \mu_{A}(x), v_{A}(x)\right\rangle \mid x \in X\right\}$, where $\mu_{A}, v_{A}: X \rightarrow[0,1]$, with the condition $0 \leq \mu_{A}(x)+v_{A}(x) \leq 1$ for all $x \in X$.

The numbers $\mu_{A}(x), v_{A}(x)$ are called the degree of membership and nonmembership of $x$ to $A$, respectively. We call $\pi_{A}(x)=1-\mu_{A}(x)-v_{A}(x)$ the intuitionistic index of $x$ in $A$, which denotes the hesitancy degree of $x$ to $A$. And it is evident that $0 \leq \pi_{A}(x) \leq 1$ for all $x \in X$. For convenience, we abbreviate the intuitionistic fuzzy set to IFS and denote the set of all IFSs on $X$ by $\operatorname{IFS}(X)$.

Sometimes, we can not give the accurate values of membership degree and nonmembership degree, but a value range. In such cases, Atanassov and Gargov [7] introduced the following notion of the interval-valued intuitionistic fuzzy set, which generalized IFS.

Definition 2 (see [7]). Let a nonempty set $X$ be the universe of discourse and let int $[0,1]$ be all closed subintervals of the interval $[0,1]$. An interval-valued intuitionistic fuzzy set is defined by Atanassov and Gargov as $A=\left\{\left\langle x, \mu_{A}(x), \nu_{A}(x)\right\rangle \mid\right.$ $x \in X\}$, where $\mu_{A}, v_{A}: X \rightarrow \operatorname{int}[0,1]$, with the condition $0 \leq$ $\sup \left(\mu_{A}(x)\right)+\sup \left(\nu_{A}(x)\right) \leq 1$ for all $x \in X$.

The interval numbers $\mu_{A}(x), v_{A}(x)$ are called the degree of membership and nonmembership of $x$ to $A$, respectively. For convenience, we denote $\mu_{A}(x)=\left[\mu_{A}^{-}(x), \mu_{A}^{+}(x)\right], v_{A}(x)=$ $\left[v_{A}^{-}(x), v_{A}^{+}(x)\right]$, and then $A=\left\{\left\langle x,\left[\mu_{A}^{-}(x), \mu_{A}^{+}(x)\right],\left[v_{A}^{-}(x)\right.\right.\right.$, $\left.\left.\left.v_{A}^{+}(x)\right]\right\rangle \mid x \in X\right\}$ with condition $0 \leq \mu_{A}^{+}(x)+v_{A}^{+}(x) \leq 1$ and call the interval $\left[1-\mu_{A}^{+}(x)-\nu_{A}^{+}(x), 1-\mu_{A}^{-}(x)-\nu_{A}^{-}(x)\right]$ abbreviated by $\left[\pi_{A}^{-}(x), \pi_{A}^{+}(x)\right]=\pi_{A}(x)$ the interval-valued intuitionistic index of $x$ in $A$, which denotes the hesitancy degree of $x$ to $A$. Similarly, we abbreviate the interval-valued intuitionistic fuzzy set to IvIFS and denote the set of all the IvIFSs on $X$ by $\operatorname{IvIFS}(X)$. 
Definition 3 (see $[18,24]$ ). Let $\left[\mu_{A}^{-}, \mu_{A}^{+}\right],\left[\mu_{B}^{-}, \mu_{B}^{+}\right] \in \operatorname{int}[0,1]$; we define
(a) $\left[\mu_{A}^{-}, \mu_{A}^{+}\right] \leq\left[\mu_{B}^{-}, \mu_{B}^{+}\right]$, iff $\mu_{A}^{-} \leq \mu_{B}^{-}, \mu_{A}^{+} \leq \mu_{B}^{+}$;
(b) $\left[\mu_{A}^{-}, \mu_{A}^{+}\right] \leq\left[\mu_{B}^{-}, \mu_{B}^{+}\right]$, iff $\mu_{A}^{-} \leq \mu_{B}^{-}, \mu_{A}^{+} \geq \mu_{B}^{+}$;
(c) $\left[\mu_{A}^{-}, \mu_{A}^{+}\right]=\left[\mu_{B}^{-}, \mu_{B}^{+}\right]$, iff $\mu_{A}^{-}=\mu_{B}^{-}, \mu_{A}^{+}=\mu_{B}^{+}$.

Definition 4 (see $[18,24])$. For two IvIFSs $A=\left\{\left\langle x,\left[\mu_{A}^{-}(x)\right.\right.\right.$, $\left.\left.\left.\mu_{A}^{+}(x)\right],\left[\nu_{A}^{-}(x), v_{A}^{+}(x)\right]\right\rangle \mid x \in X\right\}, B=\left\{\left\langle x,\left[\mu_{B}^{-}(x), \mu_{B}^{+}(x)\right]\right.\right.$, $\left.\left.\left[v_{B}^{-}(x), v_{B}^{+}(x)\right]\right\rangle \mid x \in X\right\}$, we define the following relations and operation:

(a) $A \subseteq B$, iff $\left[\mu_{A}^{-}(x), \mu_{A}^{+}(x)\right] \leq\left[\mu_{B}^{-}(x), \mu_{B}^{+}(x)\right],\left[\nu_{A}^{-}(x)\right.$, $\left.\nu_{A}^{+}(x)\right] \geq\left[\nu_{B}^{-}(x), \nu_{B}^{+}(x)\right]$, for all $x \in X$

(b) $A \preceq B$, iff $\left[\mu_{A}^{-}(x), \mu_{A}^{+}(x)\right] \preceq\left[\mu_{B}^{-}(x), \mu_{B}^{+}(x)\right],\left[v_{A}^{-}(x)\right.$, $\left.v_{A}^{+}(x)\right] \geq\left[\nu_{B}^{-}(x), v_{B}^{+}(x)\right]$, for all $x \in X$

(c) $A=B$, iff $\left[\mu_{A}^{-}(x), \mu_{A}^{+}(x)\right]=\left[\mu_{B}^{-}(x), \mu_{B}^{+}(x)\right],\left[\nu_{A}^{-}(x)\right.$, $\left.\nu_{A}^{+}(x)\right]=\left[\nu_{B}^{-}(x), \nu_{B}^{+}(x)\right]$, for all $x \in X ;$

(d) $A^{c}=\left\{\left\langle x,\left[v_{A}^{-}(x), v_{A}^{+}(x)\right],\left[\mu_{A}^{-}(x), \mu_{A}^{+}(x)\right]\right\rangle \mid x \in X\right\}$.

Definition 5 (see [9]). Let $A, B \in \operatorname{IvIFS}(X), X=\left\{x_{1}, x_{2}, \ldots\right.$, $\left.x_{n}\right\}, A=\left\{\left\langle x_{i},\left[\mu_{A}^{-}\left(x_{i}\right), \mu_{A}^{+}\left(x_{i}\right)\right],\left[v_{A}^{-}\left(x_{i}\right), v_{A}^{+}\left(x_{i}\right)\right]\right\rangle \mid x_{i} \in X\right\}$, and $B=\left\{\left\langle x_{i},\left[\mu_{B}^{-}\left(x_{i}\right), \mu_{B}^{+}\left(x_{i}\right)\right],\left[\nu_{B}^{-}\left(x_{i}\right), \nu_{B}^{+}\left(x_{i}\right)\right]\right\rangle \mid x_{i} \in X\right\}$; then the correlation coefficient of $A$ and $B$ is defined by

$$
K(A, B)=\frac{C(A, B)}{\sqrt{E(A) E(B)}},
$$

where the correlation of two IvIFSs $A$ and $B$ is given by

$$
\begin{aligned}
& C(A, B)=\frac{1}{2} \sum_{i=1}^{n}\left\{\mu_{A}^{-}\left(x_{i}\right) \mu_{B}^{-}\left(x_{i}\right)+\mu_{A}^{+}\left(x_{i}\right) \mu_{B}^{+}\left(x_{i}\right)\right. \\
& +v_{A}^{-}\left(x_{i}\right) \nu_{B}^{-}\left(x_{i}\right)+v_{A}^{+}\left(x_{i}\right) \nu_{B}^{+}\left(x_{i}\right)+\pi_{A}^{-}\left(x_{i}\right) \pi_{B}^{-}\left(x_{i}\right) \\
& \left.+\pi_{A}^{+}\left(x_{i}\right) \pi_{B}^{+}\left(x_{i}\right)\right\}
\end{aligned}
$$

and the informational intuitionistic energies of two IvIFSs $A$ and $B$ are given by

$$
\begin{gathered}
E(A)=\frac{1}{2} \sum_{i=1}^{n}\left\{\left[\mu_{A}^{-}\left(x_{i}\right)\right]^{2}+\left[\mu_{A}^{+}\left(x_{i}\right)\right]^{2}+\left[\nu_{A}^{-}\left(x_{i}\right)\right]^{2}\right. \\
\left.+\left[v_{A}^{+}\left(x_{i}\right)\right]^{2}+\left[\pi_{A}^{-}\left(x_{i}\right)\right]^{2}+\left[\pi_{A}^{+}\left(x_{i}\right)\right]^{2}\right\}, \\
E(B)=\frac{1}{2} \sum_{i=1}^{n}\left\{\left[\mu_{B}^{-}\left(x_{i}\right)\right]^{2}+\left[\mu_{B}^{+}\left(x_{i}\right)\right]^{2}+\left[\nu_{B}^{-}\left(x_{i}\right)\right]^{2}\right. \\
\left.+\left[\nu_{B}^{+}\left(x_{i}\right)\right]^{2}+\left[\pi_{B}^{-}\left(x_{i}\right)\right]^{2}+\left[\pi_{B}^{+}\left(x_{i}\right)\right]^{2}\right\} .
\end{gathered}
$$

The correlation coefficient of two IvIFSs $A$ and $B$ satisfies the following properties [9]:

(a) $0 \leq K(A, B) \leq 1$;

(b) $K(A, B)=K(B, A)$;

(c) $K(A, B)=1$ iff $A=B$.

\section{The Uncertain Information of IvIFS}

In this section, we give some kinds of entropy to measure different types of uncertain information of IvIFS and show an efficient compositive entropy measure of IvIFS. We only discuss the case where the universe has finite objects; that is, $X=\left\{x_{1}, x_{2}, \ldots, x_{n}\right\}$.

In order to describe the discrimination information of IFSs, Mao et al. [23] define a suitable intuitionistic fuzzy cross-entropy. Let $A, B \in \operatorname{IFS}(X), X=\left\{x_{1}, x_{2}, \ldots, x_{n}\right\}$; then the intuitionistic fuzzy cross-entropy of $A$ against $B$ based on uncertain information is defined as

$$
\begin{gathered}
C E(A, B)=\sum_{i=1}^{n}\left(\pi_{A}\left(x_{i}\right) \ln \frac{\pi_{A}\left(x_{i}\right)}{(1 / 2)\left(\pi_{A}\left(x_{i}\right)+\pi_{B}\left(x_{i}\right)\right)}\right. \\
\left.+\Delta_{A}\left(x_{i}\right) \ln \frac{\Delta_{A}\left(x_{i}\right)}{(1 / 2)\left(\Delta_{A}\left(x_{i}\right)+\Delta_{B}\left(x_{i}\right)\right)}\right),
\end{gathered}
$$

where $\pi_{A}\left(x_{i}\right)=1-\mu_{A}\left(x_{i}\right)-v_{A}\left(x_{i}\right)$ shows degree of hesitancy and $\Delta_{A}\left(x_{i}\right)=\left|\mu_{A}\left(x_{i}\right)-v_{A}\left(x_{i}\right)\right|$ describe the balance of power between membership degree and nonmembership degree of $x$ to $A$.

The symmetric intuitionistic fuzzy cross-entropy is $D E(A, B)=C E(A, B)+C E(B, A)$, and it has been proved that $0 \leq C E(A, B) \leq n \ln 2,0 \leq D E(A, B) \leq 2 n \ln 2$.

The symmetric intuitionistic fuzzy cross-entropy $D E(A$, $B$ ) is used to describe discrimination uncertain information which includes intuitionism and fuzziness. So when $B$ is a crisp set in $D E(A, B)$, that is, $\pi_{B}(x)=0, \Delta_{B}(x)=1$ for all $x \in X$, then $D E(A, B)$ can describe intuitionistic and fuzzy information of $A$. Based on this, Mao et al. [23] construct an intuitionistic fuzzy entropy measure

$$
\begin{aligned}
E & (A)=\frac{1}{2 n \ln 2} D E(A, B)=\frac{1}{2 n \ln 2} \sum_{i=1}^{n}\left(\pi_{A}\left(x_{i}\right) \ln 2\right. \\
& +\Delta_{A}\left(x_{i}\right) \ln \Delta_{A}\left(x_{i}\right) \\
& \left.+\left(\Delta_{A}\left(x_{i}\right)+1\right) \ln \frac{2}{\Delta_{A}\left(x_{i}\right)+1}\right)
\end{aligned}
$$

which satisfies four axiomatic principles of the intuitionistic fuzzy entropy in [23].

3.1. Interval-Valued Intuitionistic Fuzzy Cross-Entropy and Interval-Valued Intuitionistic Fuzzy Entropy. In order to reflect the intuitionism and fuzziness of IvIFS, we will introduce two factors $\Psi_{A}(x), \Delta_{A}(x)$.

Definition 6. Let $A \in \operatorname{IvIFS}(X), A=\left\{\left\langle x,\left[\mu_{A}^{-}(x), \mu_{A}^{+}(x)\right]\right.\right.$, $\left.\left.\left[v_{A}^{-}(x), v_{A}^{+}(x)\right]\right\rangle \mid x \in X\right\},\left[\pi_{A}^{-}(x), \pi_{A}^{+}(x)\right]=\left[1-\mu_{A}^{+}(x)-v_{A}^{+}(x)\right.$, $\left.1-\mu_{A}^{-}(x)-v_{A}^{-}(x)\right]$. The intuitionistic factor $\Psi_{A}(x)$ is defined as the arithmetic average of $\pi_{A}^{-}(x)$ and $\pi_{A}^{+}(x)$; that is,

$$
\begin{aligned}
\Psi_{A}(x) & =\frac{\pi_{A}^{-}(x)+\pi_{A}^{+}(x)}{2} \\
& =\frac{2-\mu_{A}^{-}(x)-\mu_{A}^{+}(x)-\nu_{A}^{-}(x)-\nu_{A}^{+}(x)}{2}
\end{aligned}
$$

which can depict the average hesitancy degree of $x$ to $A$. 
The fuzzy factor $\Delta_{A}(x)$ is defined as the distance between $\left[\mu_{A}^{-}(x), \mu_{A}^{+}(x)\right]$ and $\left[\nu_{A}^{-}(x), v_{A}^{+}(x)\right]$; that is,

$$
\Delta_{A}(x)=\frac{1}{2}\left|\mu_{A}^{-}(x)-v_{A}^{-}(x)\right|+\frac{1}{2}\left|\mu_{A}^{+}(x)-v_{A}^{+}(x)\right|
$$

which can describe the balance of power between membership degree and nonmembership degree of $x$ to $A$.

Example 7. Assume that there is an expert evaluating an event, where the support degree is given by interval $[0.6,0.8]$ and the opposition degree is the interval $[0,0.2]$; then the hesitancy degree is the interval $[0,0.4]$. We can calculate the intuitionistic factor by $\Psi=(0+0.4) / 2=0.2$ and the fuzzy factor by $\Delta=(1 / 2)|0.6-0|+(1 / 2)|0.8-0.2|=0.6$.

For a crisp set $A$, that is, $\mu_{A}(x)=[1,1], \nu_{A}(x)=[0,0]$ or $\mu_{A}(x)=[0,0], v_{A}(x)=[1,1]$ for all $x \in X$, we can calculate $\Psi_{A}(x)=0, \Delta_{A}(x)=1$ for all $x \in X$.

Now we will define the interval-valued intuitionistic fuzzy cross-entropy of IvIFS based on the intuitionistic fuzzy crossentropy of IFS [23] as follows.

Definition 8. Let $A, B \in \operatorname{IvIFS}(X), X=\left\{x_{1}, x_{2}, \ldots, x_{n}\right\}$. Based on intuitionistic factor and fuzzy factor, the interval-valued intuitionistic fuzzy cross-entropy of $A$ against $B$ is defined as

$$
\begin{gathered}
C E(A, B)=\sum_{i=1}^{n}\left(\Psi_{A}\left(x_{i}\right) \ln \frac{\Psi_{A}\left(x_{i}\right)}{(1 / 2)\left(\Psi_{A}\left(x_{i}\right)+\Psi_{B}\left(x_{i}\right)\right)}\right. \\
\left.+\Delta_{A}\left(x_{i}\right) \ln \frac{\Delta_{A}\left(x_{i}\right)}{(1 / 2)\left(\Delta_{A}\left(x_{i}\right)+\Delta_{B}\left(x_{i}\right)\right)}\right),
\end{gathered}
$$

where

$$
\begin{aligned}
\Psi_{A}\left(x_{i}\right)= & \frac{\pi_{A}^{-}\left(x_{i}\right)+\pi_{A}^{+}\left(x_{i}\right)}{2} \\
= & \frac{2-\mu_{A}^{-}\left(x_{i}\right)-\mu_{A}^{+}\left(x_{i}\right)-v_{A}^{-}\left(x_{i}\right)-v_{A}^{+}\left(x_{i}\right)}{2}, \\
\Delta_{A}\left(x_{i}\right)= & \frac{1}{2}\left|\mu_{A}^{-}\left(x_{i}\right)-v_{A}^{-}\left(x_{i}\right)\right| \\
& +\frac{1}{2}\left|\mu_{A}^{+}\left(x_{i}\right)-v_{A}^{+}\left(x_{i}\right)\right| .
\end{aligned}
$$

Proposition 9. Let $A, B \in \operatorname{IvIFS}(X) ; \operatorname{CE}(A, B)$ satisfies following properties:
(a) $C E(A, B)=C E\left(A, B^{c}\right)=C E\left(A^{c}, B\right)=C E\left(A^{c}, B^{c}\right)$;
(b) $C E(A, B)=0$ iff $A=B$ or $A=B^{c}$;
(c) $0 \leq C E(A, B) \leq n \ln 2$.

Proof. (a) One has $A^{c}=\left\{\left\langle x_{i},\left[\nu_{A}^{-}\left(x_{i}\right), v_{A}^{+}\left(x_{i}\right)\right],\left[\mu_{A}^{-}\left(x_{i}\right)\right.\right.\right.$, $\left.\left.\left.\mu_{A}^{+}\left(x_{i}\right)\right]\right\rangle \quad \mid x_{i} \in X\right\}$. Based on formulas (6) and (7) in Definition 6, we can see $\Psi_{A}\left(x_{i}\right)=\Psi_{A^{c}}\left(x_{i}\right), \Psi_{B}\left(x_{i}\right)=\Psi_{B^{c}}\left(x_{i}\right)$, $\Delta_{A}\left(x_{i}\right)=\Delta_{A^{c}}\left(x_{i}\right)$, and $\Delta_{B}\left(x_{i}\right)=\Delta_{B^{c}}\left(x_{i}\right)$ for all $x_{i} \in X$. From equation $C E(A, B)(8)$, it is easy to verify property (a). (b) First we have $0 \leq \Psi_{A}(x)+\Delta_{A}(x)=\left(2-\mu_{A}^{-}(x)-\right.$ $\left.\mu_{A}^{+}(x)-v_{A}^{-}(x)-v_{A}^{+}(x)\right) / 2+(1 / 2)\left|\mu_{A}^{-}(x)-v_{A}^{-}(x)\right|+(1 / 2) \mid \mu_{A}^{+}(x)-$ $\nu_{A}^{+}(x) \mid \leq\left(2-\mu_{A}^{-}(x)-\mu_{A}^{+}(x)-v_{A}^{-}(x)-v_{A}^{+}(x)\right) / 2+(1 / 2)\left(\mu_{A}^{-}(x)+\right.$ $\left.\nu_{A}^{-}(x)\right)+(1 / 2)\left(\mu_{A}^{+}(x)+\nu_{A}^{+}(x)\right)=1$, for all $x \in X$. According to Shannon's inequality, we can verify $C E(A, B) \geq 0$ and $C E(A, B)=0$ iff $A=B$ or $A=B^{c}$.

(c) The relation $\Psi_{A}\left(x_{i}\right) \ln \left(\Psi_{A}\left(x_{i}\right) /(1 / 2)\left(\Psi_{A}\left(x_{i}\right)+\Psi_{B}\left(x_{i}\right)\right)\right)$ $+\Delta_{A}\left(x_{i}\right) \ln \left(\Delta_{A}\left(x_{i}\right) /\left((1 / 2)\left(\Delta_{A}\left(x_{i}\right)+\Delta_{B}\left(x_{i}\right)\right)\right)\right) \leq \ln 2\left(\Psi_{A}\left(x_{i}\right)+\right.$ $\left.\Delta_{A}\left(x_{i}\right)\right) \leq \ln 2$ is true, and when $\Psi_{A}\left(x_{i}\right)=1, \Delta_{A}\left(x_{i}\right)=0$ or $\Psi_{A}\left(x_{i}\right)=0, \Delta_{A}\left(x_{i}\right)=1, \Psi_{A}\left(x_{i}\right) \ln \left(\Psi_{A}\left(x_{i}\right) /\left((1 / 2)\left(\Psi_{A}\left(x_{i}\right)+\right.\right.\right.$ $\left.\left.\left.\Psi_{B}\left(x_{i}\right)\right)\right)\right)+\Delta_{A}\left(x_{i}\right) \ln \left(\Delta_{A}\left(x_{i}\right) /\left((1 / 2)\left(\Delta_{A}\left(x_{i}\right)+\Delta_{B}\left(x_{i}\right)\right)\right)\right)$ reaches maximum $\ln 2$. For all $x_{i} \in X$, property (c) can be verified.

Now, we proceed to the symmetric interval-valued intuitionistic fuzzy cross-entropy $D E(A, B)=C E(A, B)+C E(B$, $A)$. It is easy to verify that $D E(A, B)$ have the following properties:
(a) $D E(A, B)=D E(B, A)$;
(b) $D E(A, B)=D E\left(A, B^{c}\right)=D E\left(A^{c}, B\right)=D E\left(A^{c}, B^{c}\right)$;
(c) $C E(A, B)=0$ iff $A=B$ or $A=B^{c}$;
(d) $0 \leq D E(A, B) \leq 2 n \ln 2$.

Based on intuitionistic factor and fuzzy factor of IvIFS, we propose the axiomatic conditions of the interval-valued intuitionistic fuzzy entropy, which can measure the uncertain information of fuzziness and intuitionism of IvIFS.

Definition 10. For an IvIFS $A$ defined on $X$, the intervalvalued intuitionistic fuzzy entropy is a real-valued function $E(A)=f\left(\Psi_{A}, \Delta_{A}\right): \operatorname{IvIFS}(X) \rightarrow[0,1]$, which satisfies the following axiomatic conditions:

(a) $E(A)=0$ iff $A$ is a crisp set; that is, $\mu_{A}(x)=[1,1]$, $\nu_{A}(x)=[0,0]$ or $\mu_{A}(x)=[0,0], \nu_{A}(x)=[1,1]$ for all $x \in X$;

(b) $E(A)=1$ iff $\nu_{A}(x)=\mu_{A}(x)=[0,0], \pi_{A}(x)=[1,1]$ for all $x \in X$;

(c) $E(A)=E\left(A^{c}\right)$;

(d) $E(A)=f\left(\Psi_{A}, \Delta_{A}\right)$ is a real-valued continuous function being increasing with respect to the variable $\Psi_{A}$ and decreasing with the variable $\Delta_{A}$, where $\Psi_{A}, \Delta_{A}$ are in Definition 6.

Analogously, we know when $P$ is a crisp set, that is, $\Psi_{P}(x)=0, \Delta_{P}(x)=1$ for all $x \in X, D E(A, P)$ can measure the uncertain information of IvIFS, including fuzziness and intuitionism.

Theorem 11. Let $A \in \operatorname{IvIFS}(X)$ and $P$ is a crisp set; then

$$
\begin{aligned}
& E(A)=\frac{1}{2 n \ln 2} D E(A, P)=\frac{1}{2 n \ln 2} \sum_{i=1}^{n}\left(\Psi_{A}\left(x_{i}\right) \ln 2\right. \\
& +\Delta_{A}\left(x_{i}\right) \ln \Delta_{A}\left(x_{i}\right) \\
& \left.+\left(\Delta_{A}\left(x_{i}\right)+1\right) \ln \frac{2}{\Delta_{A}\left(x_{i}\right)+1}\right)
\end{aligned}
$$


is an interval-valued intuitionistic fuzzy entropy measure, where $D E(A, P)$ is the symmetric interval-valued intuitionistic fuzzy cross-entropy.

Proof. For $E(A)$, in order to be qualified as the intervalvalued intuitionistic fuzzy entropy measure, it must satisfy conditions (a)-(d) in Definition 10.

(a) Let $A$ be a crisp set; that is, $\Psi_{A}(x)=0, \Delta_{A}(x)=1$ for all $x \in X$; it is easy to verify that $E(A)=0$. Conversely, suppose $E(A)=0$; that is, $D E(A, P)=0$; from the properties of $D E(A, B)$, we know $A=P$ or $A=P^{c}$, which means $A$ is also a crisp set.

(b) For an IvIFS $A$, when $v_{A}(x)=\mu_{A}(x)=[0,0]$, $\pi_{A}(x)=[1,1]$ for all $x \in X$, we can calculate $\Psi_{A}(x)=1$, $\Delta_{A}(x)=0$. It is easy to verify that $E(A)=1$. Conversely, when $E(A)=1$, we only have $\Psi_{A}\left(x_{i}\right) \ln 2+\Delta_{A}\left(x_{i}\right) \ln \Delta_{A}\left(x_{i}\right)+$ $\left(\Delta_{A}\left(x_{i}\right)+1\right) \ln \left(2 /\left(\Delta_{A}\left(x_{i}\right)+1\right)\right)=2 \ln 2$ iff $\Psi_{A}\left(x_{i}\right)=$ $\left(\pi_{A}^{-}\left(x_{i}\right)+\pi_{A}^{+}\left(x_{i}\right)\right) / 2=1, \Delta_{A}\left(x_{i}\right)=(1 / 2)\left|\mu_{A}^{-}\left(x_{i}\right)-v_{A}^{-}\left(x_{i}\right)\right|+$ $(1 / 2)\left|\mu_{A}^{+}\left(x_{i}\right)-v_{A}^{+}\left(x_{i}\right)\right|=0$ iff $v_{A}\left(x_{i}\right)=\mu_{A}\left(x_{i}\right)=[0,0]$, $\pi_{A}\left(x_{i}\right)=[1,1]$ for all $x_{i} \in X$.

(c) From the properties of $D E(A, B)$, we know $D E(A, P)=$ $D E\left(A^{c}, P\right)$, so $E(A)=E\left(A^{c}\right)$.

(d) In order to prove that $E(A)$ satisfies condition (d) in Definition 10, it suffices to prove that the function $f(x, y)=$ $x \ln 2+y \ln y+(y+1)[\ln 2-\ln (y+1)]$, where $x, y \in$ $[0,1]$, is increasing with its argument $x$ and decreasing for its argument $y$. Taking the partial derivative of $f$ with $x$ and $y$, respectively, we yield

$$
\begin{aligned}
& \frac{\partial f(x, y)}{\partial x}=\ln 2 \geq 0, \\
& \frac{\partial f(x, y)}{\partial y}=\ln y+\ln 2-\ln (y+1)=\ln \frac{2 y}{y+1} .
\end{aligned}
$$

Since $0 \leq y \leq 1$, we have $0 \leq 2 y /(y+1) \leq 1, \ln (2 y /(y+1)) \leq$ 0 , so $\partial f(x, y) / \partial y \leq 0$.

3.2. Interval-Value Intuitionistic Entropy and Interval-Valued Fuzzy Entropy. Mao et al. [23] presented that the intuitionistic fuzzy entropy for IFS could measure intuitionism and fuzziness of IFS. And they rewrote the intuitionistic fuzzy entropy measure as $E(A)=(1 / 2) E_{I}(A)+(1 / 2) E_{F}(A)$, where $E_{I}(A), E_{F}(A)$ are the intuitionistic entropy and fuzzy entropy measure of $A$ of IFS, respectively. For IvIFS, the intervalvalued intuitionistic fuzzy entropy has similar characteristic.

In fact, for the interval-valued intuitionistic fuzzy entropy measure $E(A)$, we have

$$
\begin{aligned}
& E(A)=\frac{1}{2 n \ln 2} \sum_{i=1}^{n}\left(\Psi_{A}\left(x_{i}\right) \ln 2+\Delta_{A}\left(x_{i}\right) \ln \Delta_{A}\left(x_{i}\right)\right. \\
& \left.+\left(\Delta_{A}\left(x_{i}\right)+1\right) \ln \frac{2}{\Delta_{A}\left(x_{i}\right)+1}\right)=\frac{1}{2 n} \sum_{i=1}^{n} \Psi_{A}\left(x_{i}\right) \\
& +\frac{1}{2 n \ln 2} \sum_{i=1}^{n}\left\{\Delta_{A}\left(x_{i}\right) \ln \Delta_{A}\left(x_{i}\right)\right. \\
& \left.+\left(\Delta_{A}\left(x_{i}\right)+1\right) \ln \frac{2}{\Delta_{A}\left(x_{i}\right)+1}\right\}=\frac{1}{2} E_{I}(A)+\frac{1}{2} \\
& \cdot E_{F}(A),
\end{aligned}
$$

where $E_{I}(A)=(1 / n) \sum_{i=1}^{n} \Psi_{A}\left(x_{i}\right)$ and $E_{F}(A)=(1 /$ $n \ln 2) \sum_{i=1}^{n}\left\{\Delta_{A}\left(x_{i}\right) \ln \Delta_{A}\left(x_{i}\right)+\left(\Delta_{A}\left(x_{i}\right)+1\right) \ln \left(2 /\left(\Delta_{A}\left(x_{i}\right)+\right.\right.\right.$ 1))\}.

Now, we will extend $E_{I}(A)$ and $E_{F}(A)$ of IFS to counterparts of IvIFS.

Definition 12. For an IvIFS $A$, the interval-valued intuitionistic entropy is a real-valued function $E_{I}(A)=g_{1}\left(\Psi_{A}\right)$ : $\operatorname{IvIFS}(X) \rightarrow[0,1]$, which satisfies the following axiomatic conditions:

(a) $E_{I}(A)=0$ iff $A$ is a fuzzy set;

(b) $E_{I}(A)=1$ iff $\nu_{A}(x)=\mu_{A}(x)=[0,0], \pi_{A}(x)=[1,1]$ for all $x \in X$;

(c) $E_{I}(A)=E_{I}\left(A^{c}\right)$;

(d) $E_{I}(A)=g_{1}\left(\Psi_{A}\right)$ is a real-valued continuous function being increasing with respect to the variable $\Psi_{A}$.

Theorem 13. Let $A \in \operatorname{IvIFS}(X)$; then

$$
E_{I}(A)=\frac{1}{n} \sum_{i=1}^{n} \Psi_{A}\left(x_{i}\right)
$$

is an interval-value intuitionistic entropy measure.

Proof. For $E_{I}(A)$, in order to be qualified as an intervalvalued intuitionistic entropy measure, it must satisfy conditions (a)-(d) in Definition 12.

(a) When $E_{I}(A)=0$, we only have for all $x_{i} \in X$, $\Psi_{A}\left(x_{i}\right)=\left(\pi_{A}^{-}\left(x_{i}\right)+\pi_{A}^{+}\left(x_{i}\right)\right) / 2=0 \Rightarrow \pi_{A}^{-}\left(x_{i}\right)=\pi_{A}^{+}\left(x_{i}\right)=$ $0 \Rightarrow \mu_{A}^{+}\left(x_{i}\right)+v_{A}^{+}\left(x_{i}\right)=1, \mu_{A}^{-}\left(x_{i}\right)+v_{A}^{-}\left(x_{i}\right)=1$. Subtracting the latter two equations, we have $\mu_{A}^{+}\left(x_{i}\right)-\mu_{A}^{-}\left(x_{i}\right)+v_{A}^{+}\left(x_{i}\right)-$ $\nu_{A}^{-}\left(x_{i}\right)=0 \Rightarrow \mu_{A}^{+}\left(x_{i}\right)=\mu_{A}^{-}\left(x_{i}\right), v_{A}^{+}\left(x_{i}\right)=\nu_{A}^{-}\left(x_{i}\right)$. Additionally, $\mu_{A}^{+}\left(x_{i}\right)+\nu_{A}^{+}\left(x_{i}\right)=1$ for all $x_{i} \in X$; we know that $A$ is a fuzzy set. Conversely, when $A$ is a fuzzy set, it is easy to verify that $\Psi_{A}\left(x_{i}\right)=\left(\pi_{A}^{-}\left(x_{i}\right)+\pi_{A}^{+}\left(x_{i}\right)\right) / 2=0$ for $x_{i} \in X$; then $E_{I}(A)=0$.

(b) When $E_{I}(A)=1$, we only have $\Psi_{A}\left(x_{i}\right)=\left(\pi_{A}^{-}\left(x_{i}\right)+\right.$ $\left.\pi_{A}^{+}\left(x_{i}\right)\right) / 2=1 \Rightarrow \pi_{A}^{-}\left(x_{i}\right)=\pi_{A}^{+}\left(x_{i}\right)=1 \Rightarrow \mu_{A}^{+}\left(x_{i}\right)+v_{A}^{+}\left(x_{i}\right)=$ $0, \mu_{A}^{-}\left(x_{i}\right)+v_{A}^{-}\left(x_{i}\right)=0 \Rightarrow \mu_{A}^{+}\left(x_{i}\right)=v_{A}^{+}\left(x_{i}\right)=\mu_{A}^{-}\left(x_{i}\right)=v_{A}^{-}\left(x_{i}\right)=$ 0 . That is to say, $v_{A}\left(x_{i}\right)=\mu_{A}\left(x_{i}\right)=[0,0], \pi_{A}\left(x_{i}\right)=[1,1]$ for all $x_{i} \in X$. Conversely, when $v_{A}\left(x_{i}\right)=\mu_{A}\left(x_{i}\right)=[0,0], \pi_{A}\left(x_{i}\right)=$ $[1,1]$ for all $x_{i} \in X$, we have $\Psi_{A}\left(x_{i}\right)=\left(\pi_{A}^{-}\left(x_{i}\right)+\pi_{A}^{+}\left(x_{i}\right)\right) / 2=1$; then $E_{I}(A)=1$.

(c) Since $\Psi_{A}\left(x_{i}\right)=\Psi_{A^{c}}\left(x_{i}\right)$ for all $x_{i} \in X, E_{I}(A)=E_{I}\left(A^{c}\right)$.

(d) Condition (d) in Definition 12 is apparent.

Definition 14. For IvIFS $A$, the interval-valued fuzzy entropy is a real-valued function $E_{F}(A)=g_{2}\left(\Delta_{A}\right): \operatorname{IvIFS}(X) \rightarrow$ $[0,1]$, which satisfies the following axiomatic conditions:

(a) $E_{F}(A)=0$ iff $A$ is a crisp set;

(b) $E_{F}(A)=1$ iff $\left[\mu_{A}^{-}(x), \mu_{A}^{+}(x)\right]=\left[\nu_{A}^{-}(x), v_{A}^{+}(x)\right]$ for all $x \in X$;

(c) $E_{F}(A)=E_{F}\left(A^{c}\right)$;

(d) $E_{F}(A)=g_{2}\left(\Delta_{A}\right)$ is a real-valued continuous function being decreasing with respect to the variable $\Delta_{A}$. 
Theorem 15. Let $A \in \operatorname{IvIFS}(X)$; then

$$
\begin{gathered}
E_{F}(A)=\frac{1}{n \ln 2} \sum_{i=1}^{n}\left\{\Delta_{A}\left(x_{i}\right) \ln \Delta_{A}\left(x_{i}\right)\right. \\
\left.+\left(\Delta_{A}\left(x_{i}\right)+1\right) \ln \frac{2}{\Delta_{A}\left(x_{i}\right)+1}\right\}
\end{gathered}
$$

is an interval-value fuzzy entropy measure.

Proof. For $E_{F}(A)$, in order to be qualified as an intervalvalued fuzzy entropy measure, it must satisfy conditions (a)(d) in Definition 14.

(a) When $E_{F}(A)=0$, we only have $\Delta_{A}\left(x_{i}\right)=$ (1/ 2) $\left|\mu_{A}^{-}\left(x_{i}\right)-v_{A}^{-}\left(x_{i}\right)\right|+(1 / 2)\left|\mu_{A}^{+}\left(x_{i}\right)-v_{A}^{+}\left(x_{i}\right)\right|=1 \Rightarrow \mid \mu_{A}^{-}\left(x_{i}\right)-$ $v_{A}^{-}\left(x_{i}\right)|=1,| \mu_{A}^{+}\left(x_{i}\right)-v_{A}^{+}\left(x_{i}\right) \mid=1 \Rightarrow \mu_{A}\left(x_{i}\right)=[1,1], v_{A}\left(x_{i}\right)=$ $[0,0]$ or $\mu_{A}\left(x_{i}\right)=[0,0], \nu_{A}\left(x_{i}\right)=[1,1]$ for all $x_{i} \in X$; that is, $A$ is a crisp set. Conversely, when $A$ is a crisp set, that is, $\Delta_{A}\left(x_{i}\right)=1$, it is easy to verify that $E_{F}(A)=0$.

(b) When $E_{F}(A)=1$, we only have $\Delta_{A}\left(x_{i}\right)=$ (1/ 2) $\left|\mu_{A}^{-}\left(x_{i}\right)-v_{A}^{-}\left(x_{i}\right)\right|+(1 / 2)\left|\mu_{A}^{+}\left(x_{i}\right)-v_{A}^{+}\left(x_{i}\right)\right|=0 \Rightarrow \mu_{A}^{-}\left(x_{i}\right)=$ $v_{A}^{-}\left(x_{i}\right), \mu_{A}^{+}\left(x_{i}\right)=v_{A}^{+}\left(x_{i}\right)$ for all $x_{i} \in X$; that is, $\left[\mu_{A}^{-}\left(x_{i}\right)\right.$, $\left.\mu_{A}^{+}\left(x_{i}\right)\right]=\left[v_{A}^{-}\left(x_{i}\right), v_{A}^{+}\left(x_{i}\right)\right]$. Conversely, when $\left[\mu_{A}^{-}\left(x_{i}\right)\right.$, $\left.\mu_{A}^{+}\left(x_{i}\right)\right]=\left[v_{A}^{-}\left(x_{i}\right), v_{A}^{+}\left(x_{i}\right)\right]$, that is, $\Delta_{A}\left(x_{i}\right)=0$, it is easy to verify that $E_{F}(A)=1$.

(c) Since $\Delta_{A}(x)=\Delta_{A^{c}}(x)$ for all $x \in X, E_{F}(A)=E_{F}\left(A^{c}\right)$.

(d) From (d) in Theorem 11, we know that condition (d) in Definition 14 is satisfied.

Based on the above analysis, the interval-valued intuitionistic fuzzy entropy measure can be seen as arithmetic average of the interval-valued intuitionistic entropy measure and the interval-valued fuzzy entropy measure.

3.3. Interval-Valued Span Entropy. In this section, we will introduce another entropy of the interval-value span entropy to measure uncertain information caused by the uncertainty of membership and nonmembership degree of IvIFS.

Here, we first put forward the axiomatic conditions of the span entropy.

Definition 16. For an IvIFS $A$, the interval-valued span entropy is a real-valued function $E_{S}(A): \operatorname{IvIFS}(X) \rightarrow[0,1]$, which satisfies the following axiomatic conditions:

(a) $E_{S}(A)=0$ iff $A$ is IFS;

(b) $E_{S}(A)=1$ iff $\mu_{A}^{+}(x)+\nu_{A}^{+}(x)=1, \mu_{A}^{-}(x)=\nu_{A}^{-}(x)=0$ for all $x \in X$;

(c) $E_{S}(A)=E_{S}\left(A^{c}\right)$;

(d) let $B \in \operatorname{IvIFS}(X), E_{S}(A) \leq E_{S}(B)$, if $\left[\mu_{A}^{-}(x), \mu_{A}^{+}(x)\right] \geq$ $\left[\mu_{B}^{-}(x), \mu_{B}^{+}(x)\right]$ and $\left[v_{A}^{-}(x), v_{A}^{+}(x)\right] \succeq\left[\nu_{B}^{-}(x), v_{B}^{+}(x)\right]$ for all $x \in X$.

Since the interval-valued span entropy is a magnitude which allows us to measure the extent of variation for the interval values of membership degree and nonmembership degree, this four conditions can be understood as follows: for an IvIFS $A$, one has the following. (a) The interval-valued span entropy is zero iff the values of membership degree and nonmembership degree are certain; that is, $A$ is IFS.

(b) When the interval-valued span entropy reaches maximum, it means that the total span of $\mu_{A}(x), v_{A}(x)$ is the largest. Since $\mu_{A}^{+}(x)+\nu_{A}^{+}(x) \leq 1$, we can intuitively understand that the interval-valued span entropy reaches maximum iff $\mu_{A}^{+}(x)+\nu_{A}^{+}(x)=1, \mu_{A}^{-}(x)=\nu_{A}^{-}(x)=0$.

(c) As $A^{c}=\left\{\left\langle x,\left[v_{A}^{-}(x), v_{A}^{+}(x)\right],\left[\mu_{A}^{-}(x), \mu_{A}^{+}(x)\right]\right\rangle \mid x \in X\right\}$, for $A$ and $A^{c}$, we know that the extent of variation for the interval values of membership degree and nonmembership degree is the same.

(d) The spans of both $\mu(x)$ and $\nu(x)$ are bigger; we know that the interval values of membership degree and nonmembership degree are more uncertain; that is, the interval-valued span entropy is bigger.

Now our goal is to give the specific interval-valued span entropy measures. To construct the entropy on IFS, Burillo and Bustince [19] introduced function $\Phi_{D}: D \rightarrow[0,1]$, where $D=\{(x, y) \in[0,1] \times[0,1] \mid x+y \leq 1\}$. Meanwhile, function $\Phi_{D}$ satisfies the following conditions:
(a) $\Phi_{D}(x, y)=1$ iff $x+y=1$;
(b) $\Phi_{D}(x, y)=0$ iff $x=y=0$;
(c) $\Phi_{D}(x, y)=\Phi_{D}(y, x)$;
(d) if $x \leq x^{\prime}$ and $y \leq y^{\prime}$, then $\Phi_{D}(x, y) \leq \Phi_{D}\left(x^{\prime}, y^{\prime}\right)$.

We can verify the following functions are $\Phi_{D}(x, y)$ abbreviated by $\Phi(x, y)$ :
(a) $\Phi(x, y)=x+y$;
(b) $\Phi(x, y)=(x+y)^{n}, n=2,3, \ldots$;
(c) $\Phi(x, y)=(x+y) e^{1-(x+y)}$;
(d) $\Phi(x, y)=(x+y) \sin ((\pi / 2)(x+y))$.

Theorem 17. For an IvIFS $A$, the real-valued function $E_{S}(A)$ : $\operatorname{IvIFS}(X) \rightarrow[0,1]$

$$
E_{S}(A)=\frac{1}{n} \sum_{i=1}^{n} \Phi\left(\Delta \mu_{A}\left(x_{i}\right), \Delta v_{A}\left(x_{i}\right)\right)
$$

is an interval-valued span entropy measure, where the function of $\Phi$ satisfies the previous four conditions $(a)-(d), \Delta \mu_{A}\left(x_{i}\right)=$ $\mu_{A}^{+}\left(x_{i}\right)-\mu_{A}^{-}\left(x_{i}\right)$ is the span of membership degree, and $\Delta v_{A}\left(x_{i}\right)=v_{A}^{+}\left(x_{i}\right)-v_{A}^{-}\left(x_{i}\right)$ is the span of nonmembership degree.

Proof. For $E_{S}(A)$, in order to be qualified as an intervalvalued span entropy measure, it must satisfy conditions (a)(d) in Definition 16.

(a) Based on condition (b) of $\Phi(x, y)$, we know $E_{S}(A)=0$ iff $\Phi\left(\Delta \mu_{A}\left(x_{i}\right), \Delta v_{A}\left(x_{i}\right)\right)=0$ iff $\Delta \mu_{A}\left(x_{i}\right)=\Delta v_{A}\left(x_{i}\right)=0$ iff $\mu_{A}^{+}\left(x_{i}\right)=\mu_{A}^{-}\left(x_{i}\right), v_{A}^{+}\left(x_{i}\right)=v_{A}^{-}\left(x_{i}\right)$ for all $x_{i} \in X$ iff $A$ is IFS.

(b) Based on condition (a) of $\Phi(x, y)$, we know $E_{S}(A)=$ 1 iff $\Phi\left(\Delta \mu_{A}\left(x_{i}\right), \Delta v_{A}\left(x_{i}\right)\right)=1$ iff $\Delta \mu_{A}\left(x_{i}\right)+\Delta v_{A}\left(x_{i}\right)=1$ iff $\mu_{A}^{+}\left(x_{i}\right)-\mu_{A}^{-}\left(x_{i}\right)+v_{A}^{+}\left(x_{i}\right)-v_{A}^{-}\left(x_{i}\right)=1$ iff $\mu_{A}^{+}\left(x_{i}\right)+v_{A}^{+}\left(x_{i}\right)=1$ and $\mu_{A}^{-}\left(x_{i}\right)=v_{A}^{-}\left(x_{i}\right)=0$ for all $x_{i} \in X$.

(c) One has $A^{c}=\left\{\left\langle x,\left[\nu_{A}^{-}(x), v_{A}^{+}(x)\right],\left[\mu_{A}^{-}(x), \mu_{A}^{+}(x)\right]\right\rangle \mid\right.$ $x \in X\}$. We know $\Delta \mu_{A^{c}}\left(x_{i}\right)=\Delta v_{A}\left(x_{i}\right), \Delta v_{A^{c}}\left(x_{i}\right)=\Delta \mu_{A}\left(x_{i}\right)$. From condition (c) of $\Phi(x, y)$, we can obtain $E_{S}(A)=E_{S}\left(A^{c}\right)$. 
(d) If $\left[\mu_{A}^{-}\left(x_{i}\right), \mu_{A}^{+}\left(x_{i}\right)\right] \geq\left[\mu_{B}^{-}\left(x_{i}\right), \mu_{B}^{+}\left(x_{i}\right)\right]$ and $\left[v_{A}^{-}\left(x_{i}\right)\right.$, $\left.v_{A}^{+}\left(x_{i}\right)\right] \geq\left[\nu_{B}^{-}\left(x_{i}\right), \nu_{B}^{+}\left(x_{i}\right)\right]$, we can obtain $\Delta \mu_{A}\left(x_{i}\right) \leq \Delta \mu_{B}\left(x_{i}\right)$ and $\Delta v_{A}\left(x_{i}\right) \leq \Delta v_{B}\left(x_{i}\right)$ for all $x_{i} \in X$. From condition (d) of $\Phi(x, y)$, we know that condition (d) in Definition 16 is suited.

Using the previous function formulas of $\Phi(x, y)$, we can construct the following interval-valued span entropy measures:

(a) $E_{S}(A)=(1 / n) \sum_{i=1}^{n}\left(\mu_{A}^{+}\left(x_{i}\right)-\mu_{A}^{-}\left(x_{i}\right)+\nu_{A}^{+}\left(x_{i}\right)-v_{A}^{-}\left(x_{i}\right)\right)$;

(b) $E_{S}(A)=(1 / n) \sum_{i=1}^{n}\left(\mu_{A}^{+}\left(x_{i}\right)-\mu_{A}^{-}\left(x_{i}\right)+v_{A}^{+}\left(x_{i}\right)-v_{A}^{-}\left(x_{i}\right)\right)^{n}$, $n=2,3, \ldots$

(c) $E_{S}(A)=(1 / n) \sum_{i=1}^{n}\left(\mu_{A}^{+}\left(x_{i}\right)-\mu_{A}^{-}\left(x_{i}\right)+v_{A}^{+}\left(x_{i}\right)-\right.$ $\left.v_{A}^{-}\left(x_{i}\right)\right) e^{1-\left(\mu_{A}^{+}\left(x_{i}\right)-\mu_{A}^{-}\left(x_{i}\right)+v_{A}^{+}\left(x_{i}\right)-v_{A}^{-}\left(x_{i}\right)\right)}$;

(d) $E_{S}(A)=(1 / n) \sum_{i=1}^{n}\left(\mu_{A}^{+}\left(x_{i}\right)-\mu_{A}^{-}\left(x_{i}\right)+\nu_{A}^{+}\left(x_{i}\right)-\right.$ $\left.v_{A}^{-}\left(x_{i}\right)\right) \sin \left((\pi / 2)\left(\mu_{A}^{+}\left(x_{i}\right)-\mu_{A}^{-}\left(x_{i}\right)+v_{A}^{+}\left(x_{i}\right)-v_{A}^{-}\left(x_{i}\right)\right)\right)$.

3.4. The Compositive Entropy of IvIFS. We already know that the proposed interval-valued intuitionistic fuzzy entropy in Theorem 11 can only express fuzziness and intuitionism of IvIFS and the interval-valued span entropy can reflect another kind of uncertain information of IvIFS. Naturally, we want to propose a sensible entropy of IvIFS which can be concerned with interval-valued intuitionistic entropy, intervalvalued fuzzy entropy, and interval-valued span entropy. First, we give another type of uncertainty factor.

Definition 18. Let $A \in \operatorname{IvIFS}(X), A=\left\{\left\langle x,\left[\mu_{A}^{-}(x), \mu_{A}^{+}(x)\right]\right.\right.$, $\left.\left.\left[v_{A}^{-}(x), v_{A}^{+}(x)\right]\right\rangle \mid x \in X\right\}$. The span factor $\Upsilon_{A}(x)$ is defined as

$$
\Upsilon_{A}(x)=\mu_{A}^{+}(x)-\mu_{A}^{-}(x)+\nu_{A}^{+}(x)-\nu_{A}^{-}(x)
$$

which can depict the extent of variation for the interval values of membership degree and nonmembership degree of $x$ to $A$.

Based on intuitionistic factor, fuzzy factor, and span factor, in the following, we will give the axiomatic definition of a kind of compositive entropy.

Definition 19. For an IvIFS $A$, the compositive entropy of IvIFS also called interval-valued intuitionistic fuzzy span entropy is a real-valued function $\bar{E}(A)=h\left(\Psi_{A}, \Delta_{A}, \Upsilon_{A}\right)$ : $\operatorname{IvIFS}(X) \rightarrow[0,1]$, which satisfies the following axiomatic conditions:

(a) $\bar{E}(A)=0$ iff $A$ is a crisp set;

(b) $\bar{E}(A)=1$ iff $\mu_{A}(x)=[0,0.5], \nu_{A}(x)=[0,0.5]$ for all $x \in X$;

(c) $\bar{E}(A)=\bar{E}\left(A^{c}\right)$;

(d) $\bar{E}(A)=h\left(\Psi_{A}, \Delta_{A}, \Upsilon_{A}\right)$ is a real-valued continuous function being increasing with respect to the variables $\Psi_{A}, \Upsilon_{A}$ and decreasing with the variable $\Delta_{A}$.

$\Psi_{A}, \Delta_{A}$ are the intuitionistic factor and fuzzy factor in Definition 6 and $\Upsilon_{A}(x)$ is the span factor in Definition 18.
Inspired by equation $E(A)=(1 / 2) E_{I}(A)+(1 / 2) E_{F}(A)$, now we will construct a compositive entropy measure.

Theorem 20. Consider $A \in \operatorname{IvIFS}(X)$; then

$$
\begin{aligned}
& \bar{E}(A)=\frac{2}{5} E_{I}(A)+\frac{2}{5} E_{F}(A)+\frac{2}{5} E_{S}(A)=\frac{2}{5 n \ln 2} \\
& \cdot \sum_{i=1}^{n}\left(\left(\Psi_{A}\left(x_{i}\right)+\Upsilon_{A}\left(x_{i}\right)\right) \ln 2+\Delta_{A}\left(x_{i}\right) \ln \Delta_{A}\left(x_{i}\right)\right. \\
& \left.+\left(\Delta_{A}\left(x_{i}\right)+1\right) \ln \frac{2}{\Delta_{A}\left(x_{i}\right)+1}\right)
\end{aligned}
$$

is a compositive entropy measure of IvIFS, which is also called the interval-valued intuitionistic fuzzy span entropy measure.

Proof. For $\bar{E}(A)$, in order to be qualified as an interval-valued intuitionistic fuzzy span entropy measure, it must satisfy conditions (a)-(d) in Definition 19.

(a) It is easy to verify that $\bar{E}(A)=0$ iff $E_{I}(A)=E_{F}(A)=$ $E_{S}(A)=0$ iff $A$ is a crisp set.

(b) Taking $\Psi_{A}, \Delta_{A}$, and $\Upsilon_{A}$ into $\bar{E}(A)$, we have

$$
\begin{gathered}
\bar{E}(A)=\frac{2}{5} E_{I}(A)+\frac{2}{5} E_{F}(A)+\frac{2}{5} E_{S}(A)=\frac{2}{5 n \ln 2} \\
\cdot \sum_{i=1}^{n}\left(\left(\Psi_{A}\left(x_{i}\right)+\Upsilon_{A}\left(x_{i}\right)\right) \ln 2+\Delta_{A}\left(x_{i}\right) \ln \Delta_{A}\left(x_{i}\right)\right. \\
\left.+\left(\Delta_{A}\left(x_{i}\right)+1\right) \ln \frac{2}{\Delta_{A}\left(x_{i}\right)+1}\right)=\frac{2}{5 n \ln 2} \\
\cdot \sum_{i=1}^{n}\left(\frac{2+\mu_{A}^{+}\left(x_{i}\right)+v_{A}^{+}\left(x_{i}\right)-3 \mu_{A}^{-}\left(x_{i}\right)-3 v_{A}^{-}\left(x_{i}\right)}{2}\right. \\
\cdot \ln 2+\Delta_{A}\left(x_{i}\right) \ln \Delta_{A}\left(x_{i}\right)+\left(\Delta_{A}\left(x_{i}\right)+1\right) \\
\left.\cdot \ln \frac{2}{\Delta_{A}\left(x_{i}\right)+1}\right) .
\end{gathered}
$$

We want to prove $\bar{E}(A)=1$ iff $\mu_{A}(x)=[0,0.5], v_{A}(x)=$ $[0,0.5]$ for all $x \in X$. Sufficient condition is obvious. As for necessary condition, we can see $\left(\left(2+\mu_{A}^{+}\left(x_{i}\right)+v_{A}^{+}\left(x_{i}\right)-3 \mu_{A}^{-}\left(x_{i}\right)-\right.\right.$ $\left.\left.3 v_{A}^{-}\left(x_{i}\right)\right) / 2\right) \ln 2$ reaches maximum (3/2) $\ln 2$ when $\mu_{A}^{+}\left(x_{i}\right)+$ $v_{A}^{+}\left(x_{i}\right)=1, \mu_{A}^{-}\left(x_{i}\right)=v_{A}^{-}\left(x_{i}\right)=0$ and $\Delta_{A}\left(x_{i}\right) \ln \Delta_{A}\left(x_{i}\right)+$ $\left(\Delta_{A}\left(x_{i}\right)+1\right) \ln \left(2 /\left(\Delta_{A}\left(x_{i}\right)+1\right)\right)$ reaches maximum $\ln 2$ when $\Delta_{A}\left(x_{i}\right)=(1 / 2)\left|\mu_{A}^{-}\left(x_{i}\right)-v_{A}^{-}\left(x_{i}\right)\right|+(1 / 2)\left|\mu_{A}^{+}\left(x_{i}\right)-v_{A}^{+}\left(x_{i}\right)\right|=0$. Ultimately, we have $\mu_{A}(x)=[0,0.5], v_{A}(x)=[0,0.5]$ for all $x \in X$ when $\bar{E}(A)=1$.

(c) Since $E_{I}(A)=E_{I}\left(A^{c}\right), E_{F}(A)=E_{F}\left(A^{c}\right)$, and $E_{S}(A)=$ $E_{S}\left(A^{c}\right)$, it is easy to verify $\bar{E}(A)=\bar{E}\left(A^{c}\right)$.

(d) Considering the function: $f(x, y, z)=(x+z) \ln 2+$ $y \ln y+(y+1)[\ln 2-\ln (y+1)]$, where $x, y, z \in[0,1]$, similar to the process of proof in Theorem 11(d), we can verify that $f(x, y, z)$ is increasing with the arguments $x, z$ and decreasing with its argument $y$.

Let us now consider an IvIFS $A$ in $X=\{x\}$. Using the color of each point $\left(\Psi_{A}(x), \Upsilon_{A}(x), \Delta_{A}(x)\right)$ to represent 


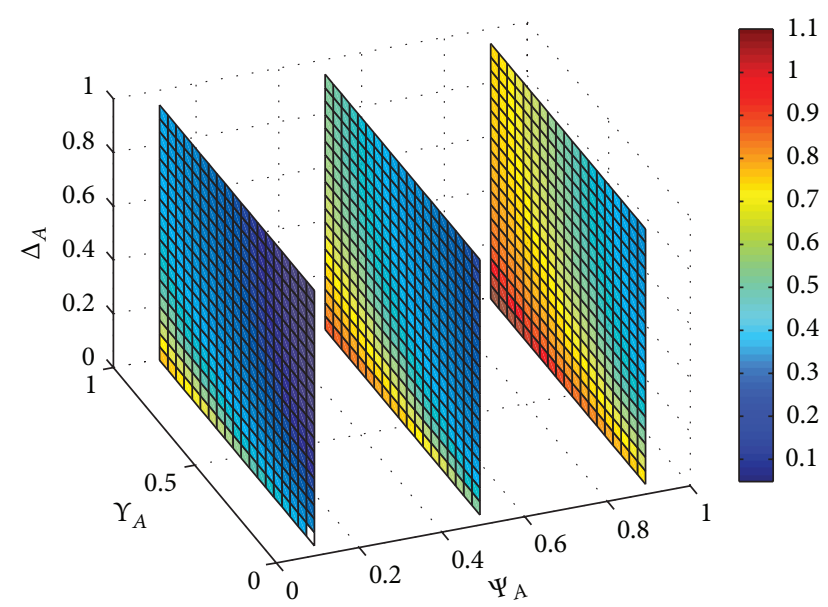

Figure 1: The compositive entropy with $\Psi_{A}=0.1,0.5,0.9$.

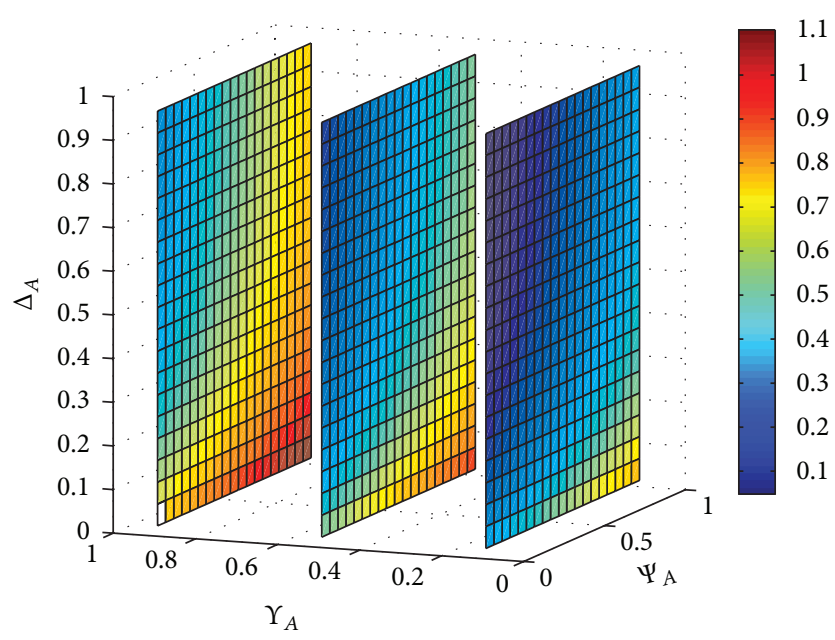

FIgURE 2: The compositive entropy with $\Upsilon_{A}=0.1,0.5,0.9$.

the value of compositive entropy, Figures 1, 2, and 3 can reflect the relation between the value of compositive entropy and $\Psi_{A}$, $\Upsilon_{A}$, and $\Delta_{A}$.

\section{The Application of the Compositive Entropy Measure}

4.1. The Effectiveness of the Compositive Entropy Measure. In fact, there have been some research results about the entropy of IvIFS; for example, Zhang et al. [24] defined $E_{1}(A)$, Wei et al. [25] put forward $E_{2}(A)$, Jin et al. [27] presented $E_{3}(A)$, Ye [28] gave $E_{4}(A)$, Zhang et al. [26] proposed $E_{5}(A)$, and Qu et al. [29] showed $E_{6}(A)$, which can be listed as follows:

(a) $E_{1}(A)=(1 / n) \sum_{i=1}^{n}\left(\left(\mu_{A}^{-}\left(x_{i}\right) \wedge v_{A}^{-}\left(x_{i}\right)+\mu_{A}^{+}\left(x_{i}\right) \wedge\right.\right.$ $\left.\left.v_{A}^{+}\left(x_{i}\right)\right) /\left(\mu_{A}^{-}\left(x_{i}\right) \vee v_{A}^{-}\left(x_{i}\right)+\mu_{A}^{+}\left(x_{i}\right) \vee v_{A}^{+}\left(x_{i}\right)\right)\right)$;

(b) $E_{2}(A)=(1 / n) \sum_{i=1}^{n}\left(\left(\mu_{A}^{-}\left(x_{i}\right) \wedge \nu_{A}^{-}\left(x_{i}\right)+\mu_{A}^{+}\left(x_{i}\right) \wedge \nu_{A}^{+}\left(x_{i}\right)+\right.\right.$ $\left.\pi_{A}^{-}\left(x_{i}\right)+\pi_{A}^{+}\left(x_{i}\right)\right) /\left(\mu_{A}^{-}\left(x_{i}\right) \vee \nu_{A}^{-}\left(x_{i}\right)+\mu_{A}^{+}\left(x_{i}\right) \vee v_{A}^{+}\left(x_{i}\right)+\pi_{A}^{-}\left(x_{i}\right)+\right.$ $\left.\left.\pi_{A}^{+}\left(x_{i}\right)\right)\right)$;

(c) $E_{3}(A)=(1 / n) \sum_{i=1}^{n}\left(\left(F_{Q}\left(\mu_{A}\left(x_{i}\right)\right) \wedge F_{Q}\left(\nu_{A}\left(x_{i}\right)\right)+1-\right.\right.$ $\left.F_{Q}\left(\mu_{A}\left(x_{i}\right)\right)-F_{Q}\left(\nu_{A}\left(x_{i}\right)\right)\right) /\left(F_{Q}\left(\mu_{A}\left(x_{i}\right)\right) \vee F_{Q}\left(\nu_{A}\left(x_{i}\right)\right)+1-\right.$ $\left.\left.F_{Q}\left(\mu_{A}\left(x_{i}\right)\right)-F_{Q}\left(\nu_{A}\left(x_{i}\right)\right)\right)\right)$, where $F_{Q}\left(\mu_{A}\left(x_{i}\right)\right)=\lambda \mu_{A}^{+}\left(x_{i}\right)+$

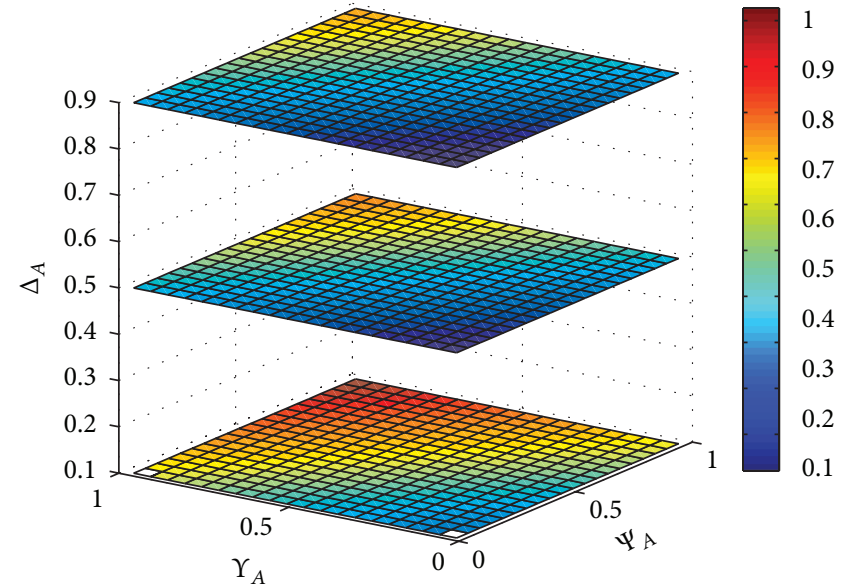

FIGURE 3: The compositive entropy with $\Delta_{A}=0.1,0.5,0.9$.

$(1-\lambda) \mu_{A}^{-}\left(x_{i}\right), F_{Q}\left(v_{A}\left(x_{i}\right)\right)=\lambda \nu_{A}^{+}\left(x_{i}\right)+(1-\lambda) \nu_{A}^{-}\left(x_{i}\right) . \lambda$ is the attitudinal character of BUM function $Q$ with $\lambda \in[0,1]$;

(d) $E_{4}(A)=(1 / n(\sqrt{2}-1)) \sum_{i=1}^{n}\left\{\sin \left(\left(\pi \times\left[1+\mu_{A}^{-}\left(x_{i}\right)+\right.\right.\right.\right.$ $\left.\left.\left.p \Delta \mu_{A}\left(x_{i}\right)-v_{A}^{-}\left(x_{i}\right)-q \Delta v_{A}\left(x_{i}\right)\right]\right) / 4\right)+\sin \left(\left(\pi \times\left[1-\mu_{A}^{-}\left(x_{i}\right)-\right.\right.\right.$ $\left.\left.\left.\left.p \Delta \mu_{A}\left(x_{i}\right)+v_{A}^{-}\left(x_{i}\right)+q \Delta v_{A}\left(x_{i}\right)\right]\right) / 4\right)-1\right\}$, where $p, q \in[0,1]$ and here we choose $p=q=0.5$;

(e) $E_{5}(A)=(1 / n) \sum_{i=1}^{n}\left(1-\left(\bar{\mu}_{A}\left(x_{i}\right)+\bar{\nu}_{A}\left(x_{i}\right)\right)\right) e^{1-\left(\bar{\mu}_{A}\left(x_{i}\right)+\bar{\nu}_{A}\left(x_{i}\right)\right)}$, where $\bar{\mu}_{A}\left(x_{i}\right)=\mu_{A}^{-}\left(x_{i}\right)+\tau \Delta \mu_{A}\left(x_{i}\right), \bar{v}_{A}\left(x_{i}\right)=\nu_{A}^{-}\left(x_{i}\right)+$ $\tau \Delta v_{A}\left(x_{i}\right), \tau \in[0,1]$. Here we choose $\tau=0.5$;

(f) $E_{6}(A)=(1 / n) \sum_{i=1}^{n}\left\{\left(\pi_{A}^{-}\left(x_{i}\right)+\pi_{A}^{+}\left(x_{i}\right)\right) / 2+\left(\left[\pi_{A}^{+}\left(x_{i}\right)\right]^{2}-\right.\right.$ $\left.\left[\pi_{A}^{-}\left(x_{i}\right)\right]^{2}\right) / 4+\left(\left[\mu_{A}^{+}\left(x_{i}\right)\right]^{2}-\left[\mu_{A}^{-}\left(x_{i}\right)\right]^{2}\right) / 4+\left(\left[\nu_{A}^{+}\left(x_{i}\right)\right]^{2}-\right.$ $\left.\left.\left[\nu_{A}^{-}\left(x_{i}\right)\right]^{2}\right) / 4\right\}$.

We will compare these entropy measures with our proposed entropy measures, including the interval-valued intuitionistic fuzzy entropy measure $E(A)$ and the compositive entropy measure $\bar{E}(A)$, by an example.

Example 21. Consider $A, B, C, D \in \operatorname{IvIFS}(X), X=\{x\}$. For short, we denote $A=\left\langle\mu_{A}(x), \nu_{A}(x), \pi_{A}(x)\right\rangle=\langle[0,0.5],[0$, $0.5],[0,1]\rangle, B=\left\langle\mu_{B}(x), \nu_{B}(x), \pi_{B}(x)\right\rangle=\langle[0.2,0.3],[0.2,0.3]$, $[0.4,0.6]\rangle, C=\left\langle\mu_{C}(x), v_{C}(x), \pi_{C}(x)\right\rangle=\langle[0.4,0.5],[0.4,0.5]$, $[0,0.2]\rangle$, and $D=\left\langle\mu_{D}(x), \nu_{D}(x), \pi_{D}(x)\right\rangle=\langle[0.4,0.5],[0,0.1]$, $[0.4,0.6]\rangle$. Based on intuitionistic factor, fuzzy factor, and span factor of IvIFS, we can analyze the uncertain relationships of $A, B$, and $C$ as follows: comparing $A$ with $B$, intuitionistic factor and fuzzy factor of $A, B$ are the same, but span factor of $A$ has a greater impact on uncertainty than $B$, so we have $\bar{E}(A)>\bar{E}(B)$. For $B$ and $C$, fuzzy factor and span factor of $B$ and $C$ are the same, but intuitionistic factor of $B$ has a greater impact on uncertainty than $C$, so we have $\bar{E}(B)>\bar{E}(C)$. Analogously, for $C$ and $D$, considering fuzzy factor, we have $\bar{E}(C)>\bar{E}(D)$. So we have the relation $\bar{E}(A)>\bar{E}(B)>\bar{E}(C)>\bar{E}(D)$.

According to entropy formulas above, we can obtain calculation results presented in Table 1.

Based on the results of Table 1, we can see only $\bar{E}$ meets the relation $\bar{E}(A)>\bar{E}(B)>\bar{E}(C)>\bar{E}(D)$. In addition, four entropy values of $A, B, C$, and $D$ only calculated by 
TABLE 1: The comparison of different entropy measures.

\begin{tabular}{ccccccccc}
\hline & $E_{1}$ & $E_{2}$ & $E_{3}$ & $E_{4}$ & $E_{5}$ & $E_{6}$ & $E$ & $\bar{E}$ \\
\hline$A$ & 1 & 1 & 1 & 1 & 0.8244 & 0.875 & 0.75 & 1 \\
$B$ & 1 & 1 & 1 & 1 & 0.8244 & 0.575 & 0.75 & 0.68 \\
$C$ & 1 & 1 & 1 & 1 & 0.1105 & 0.155 & 0.75 & 0.52 \\
$D$ & 0.1111 & 0.5789 & $\frac{5+\lambda}{9+\lambda}$ & 0.8329 & 0.8244 & 0.575 & 0.3458 & 0.3566 \\
\hline
\end{tabular}

$\bar{E}$ are different, which means only $\bar{E}$ can distinguish $A, B$, $C$, and $D$ well. Although it is possible that two or more IvIFSs have the same entropy, the proposed entropy measure is more precise and detailed than other entropy measures for presenting the degree of the uncertain information under the new framework of Definition 19.

\subsection{The Application of the Compositive Entropy Measure to} Decision-Making. Since the compositive entropy measure $\bar{E}(A)$ not only has theoretical advantage, which is taking three kinds of entropy measures into account, but also has practical excellent effect, now we will show the application on multiple attributes' decision-making with unknown information of criteria weights for alternatives.

Let $C=\left\{C_{1}, C_{2}, \ldots, C_{n}\right\}$ be a set of criteria and let $A=$ $\left\{A_{1}, A_{2}, \ldots, A_{m}\right\}$ be a set of alternatives which consists of $m$ noninferior alternatives (an alternative is noninferior if there exists no other alternative which can yield an improvement in one attribute, without causing a degradation in another). The evaluation value of criteria on an alternative $A_{i}$ is represented by the following IvIFS:

$$
\begin{gathered}
A_{i}=\left\{\left\langleC_{j},\left[\mu_{A_{i}}^{-}\left(C_{j}\right), \mu_{A_{i}}^{+}\left(C_{j}\right)\right],\right.\right. \\
\left.\left.\quad\left[v_{A_{i}}^{-}\left(C_{j}\right), v_{A_{i}}^{+}\left(C_{j}\right)\right]\right\rangle \mid C_{j} \in C\right\},
\end{gathered}
$$

where $\mu_{A_{i}}^{+}\left(C_{j}\right)+v_{A_{i}}^{+}\left(C_{j}\right) \leq 1, \mu_{A_{i}}^{-}\left(C_{j}\right), v_{A_{i}}^{-}\left(C_{j}\right) \geq 0, i=1,2, \ldots$, $m, j=1,2, \ldots, n$. For convenience, we denote $\left\langle\left[\mu_{A_{i}}^{-}\left(C_{j}\right)\right.\right.$,
$\left.\left.\mu_{A_{i}}^{+}\left(C_{j}\right)\right],\left[\nu_{A_{i}}^{-}\left(C_{j}\right), v_{A_{i}}^{+}\left(C_{j}\right)\right]\right\rangle=\left\langle\left[\mu_{i j}^{-}, \mu_{i j}^{+}\right],\left[v_{i j}^{-}, v_{i j}^{+}\right]\right\rangle=r_{i j}, i=$ $1,2, \ldots, m, j=1,2, \ldots, n$. Then the decision matrix is $R=$ $\left(r_{i j}\right)_{m \times n}$. Assume that $W=\left(\omega_{1}, \omega_{2}, \ldots, \omega_{n}\right)$ is the weight vector on attribute vector $C=\left(C_{1}, C_{2}, \ldots, C_{n}\right)$, where $\sum_{1}^{n} \omega_{i}=$ 1 and $\omega_{i} \in[0,1]$. In what follows, we only discuss the case where the weight vector of attributes $W$ is completely unknown.

According to the entropy theory [34], if the entropy value for a criterion is smaller across alternatives, it should provide decision-makers with the useful information. Therefore, the criterion should be assigned a bigger weight; otherwise, such a criterion will be judged unimportant by the decision-maker. In other words, such a criterion should be evaluated as a very small weight. Now we can establish an exact model of entropy weights [34]:

$$
\omega_{j}=\frac{1-\bar{E}\left(C_{j}\right)}{n-\sum_{i=1}^{n} \bar{E}\left(C_{i}\right)}
$$

where $\sum_{1}^{n} \omega_{i}=1$ and $\omega_{i} \in[0,1]$ and $\bar{E}\left(C_{j}\right)$ is calculated by

$$
\bar{E}\left(C_{j}\right)=\frac{1}{m} \sum_{i=1}^{m} \bar{E}\left(r_{i j}\right)
$$

where $\bar{E}\left(r_{i j}\right)$ is the compositive entropy of the IvIFS $r_{i j}$ in (17).

In multiple attributes' decision-making environment, the concept of positive point has been used to identify the best alternative. Although the positive alternative does not exist in real world, it does provide a useful theoretical evaluating standard for all the criteria. Here we define the positive alternative $A^{+}=\left\{\left\langle C_{j},[1,1],[0,0]\right\rangle \mid C_{j} \in C\right\}$. Then based on the correlation coefficient equation (1) between IvIFSs and the weight vector $W$ on attribute vector, we can define the weighted correlation coefficient $K_{W}\left(A_{i}, A^{+}\right), i=1,2, \ldots, m$, between an alternative $A_{i}$ and the positive alternative $A^{+}$as follows:

$$
K_{W}\left(A_{i}, A^{+}\right)=\frac{C_{W}\left(A_{i}, A^{+}\right)}{\sqrt{E_{W}\left(A_{i}\right) E_{W}\left(A^{+}\right)}}=\frac{(1 / 2) \sum_{j=1}^{n} \omega_{j}\left(\mu_{i j}^{-}+\mu_{i j}^{+}\right)}{\sqrt{(1 / 2) \sum_{j=1}^{n} \omega_{j}\left[\left(\mu_{i j}^{-}\right)^{2}+\left(\mu_{i j}^{+}\right)^{2}+\left(v_{i j}^{-}\right)^{2}+\left(\nu_{i j}^{+}\right)^{2}+\left(\pi_{i j}^{-}\right)^{2}+\left(\pi_{i j}^{+}\right)^{2}\right]}} .
$$

We can check that the weighted correlation coefficient $K_{W}\left(A_{i}, A^{+}\right)$has the following properties:
(a) $0 \leq K_{W}\left(A_{i}, A^{+}\right) \leq 1$;
(b) $K_{W}\left(A_{i}, A^{+}\right)=K_{W}\left(A^{+}, A_{i}\right)$;
(c) $K_{W}\left(A_{i}, A^{+}\right)=1$ iff $A_{i}=A^{+}$.

The larger the value of weighted correlation coefficient $K_{W}\left(A_{i}, A^{+}\right)$, the better the alternative $A_{i}$, as the alternative $A_{i}$ is closer to the ideal alternative $A^{+}$. Therefore, all the alternatives can be ranked according to the value of the weighted correlation coefficients so that the best alternative can be selected.

The decision procedure for the proposed method can be summarized as follows.

Step 1. Calculate the weight vector $W=\left(\omega_{1}, \omega_{2}, \ldots, \omega_{n}\right)$ on attribute vector by using (20) and (21) from the decision matrix $R=\left(r_{i j}\right)_{m \times n}$. 
Step 2. Calculate the weighted correlation coefficient $K_{W}\left(A_{i}, A^{+}\right)$by using $(22)$.

Step 3. Rank the alternatives according to the obtained correlation coefficients, and then obtain the best choice.

Example 22. Assume that a fund manager in a wealth management firm is assessing four potential investment opportunities; that is, the set of alternatives is $A\left(A_{1}, A_{2}\right.$, $\left.A_{3}, A_{4}\right)$. The firm mandates that the fund manager has to evaluate each investment against four criteria: risk $\left(C_{1}\right)$, growth $\left(C_{2}\right)$, sociopolitical issues $\left(C_{3}\right)$, and environmental impacts $\left(C_{4}\right)$. In addition, the fund manager is only comfortable with providing his/her assessment of each alternative on each criterion as an IVIFS and the decision matrix is as follows:

$$
\left[\begin{array}{llll}
([0.4,0.5],[0.3,0.4]) & ([0.4,0.6],[0.2,0.4]) & ([0.3,0.4],[0.4,0.5]) & ([0.5,0.6],[0.1,0.3]) \\
([0.5,0.6],[0.2,0.3]) & ([0.6,0.7],[0.2,0.3]) & ([0.5,0.6],[0.3,0.4]) & ([0.4,0.7],[0.1,0.2]) \\
([0.3,0.5],[0.3,0.4]) & ([0.1,0.3],[0.5,0.6]) & ([0.2,0.5],[0.4,0.5]) & ([0.2,0.3],[0.4,0.6]) \\
([0.2,0.5],[0.3,0.4]) & ([0.4,0.7],[0.1,0.2]) & ([0.4,0.5],[0.3,0.5]) & ([0.5,0.8],[0.1,0.2])
\end{array}\right] .
$$

Each element of this matrix is an IvIFS, representing the fund managers assessment as to what degree an alternative is and is not an excellent investment as per criterion. For instance, the top-left cell, $([0.4,0.5],[0.3,0.4])$, reflects the fund managers belief that alternative $A_{1}$ is an excellent investment from a risk perspective $\left(C_{1}\right)$ with a margin of $40-$ $50 \%$ and $A_{1}$ is not an excellent choice given its risk profile $\left(C_{1}\right)$ with a chance between $30 \%$ and $40 \%$. The proposed method is applied to solve this problem according to the following computational procedure.

Step 1. We can calculate the entropy weights of criteria by using (20) and (21) from the decision matrix as follows:

$$
\begin{aligned}
& \omega_{1}=0.2266, \\
& \omega_{2}=0.2722, \\
& \omega_{3}=0.2333, \\
& \omega_{4}=0.2679 .
\end{aligned}
$$

Step 2. Calculate the weighted correlation coefficient $K_{W}\left(A_{i}, A^{+}\right)$by using (22) as follows:

$$
\begin{aligned}
& K_{W}\left(A_{1}, A^{+}\right)=0.7273, \\
& K_{W}\left(A_{2}, A^{+}\right)=0.8441, \\
& K_{W}\left(A_{3}, A^{+}\right)=0.4553, \\
& K_{W}\left(A_{4}, A^{+}\right)=0.7438 .
\end{aligned}
$$

Step 3. From the weighted correlation coefficients between the alternatives and the positive alternative, the ranking order is $A_{2} \geq A_{4} \geq A_{1} \geq A_{3}$. And the alternative $A_{2}$ is the best choice.

Wei [35] and Zhang et al. [26] have introduced two kinds of efficient methods for multiple attribute decision-making with completely unknown attribute weights. By applying Wei's method [35] to Example 22, the ranking order of all the alternatives is $A_{2} \geq A_{4} \geq A_{1} \geq A_{3}$, and the most desirable alternative is $A_{2}$. By applying the method proposed by Zhang et al. [26] to Example 22, the ranking order of all the alternatives is $A_{2} \geq A_{4} \geq A_{1} \geq A_{3}$, and the most desirable alternative is $A_{2}$. We can see that all results are uniform.

4.3. The Application of the Compositive Entropy Measure to Pattern Recognition. First, we present a similarity measure of IvIFS via the relationship between similarity measure and entropy of IvIFS. Hu and Li [36] give transformation methods from entropy to similarity of IvIFS. Utilizing the compositive entropy measure above, we propose a new similarity measure which can be used for pattern recognition.

For $A, B \in \operatorname{IvIFS}(X)$, we define $f(A, B) \in \operatorname{IvIFS}(X)$ as follows:

$$
\begin{aligned}
\mu_{f(A, B)}(x) & =\left[\mu_{f(A, B)}^{-}(x), \mu_{f(A, B)}^{+}(x)\right], \\
v_{f}(A, B)(x) & =\left[\nu_{f(A, B)}^{-}(x), \nu_{f(A, B)}^{+}(x)\right],
\end{aligned}
$$

$\forall x \in X$

where

$$
\begin{aligned}
& \mu_{f(A, B)}^{-}(x) \\
& \quad=\frac{1+\left[\left|\mu_{A}^{-}(x)-\mu_{B}^{-}(x)\right| \vee\left|\nu_{A}^{+}(x)-v_{B}^{+}(x)\right|\right]^{3}}{2}, \\
& \mu_{f(A, B)}^{+}(x) \\
& \quad=\frac{1+\left[\left|\mu_{A}^{-}(x)-\mu_{B}^{-}(x)\right| \vee\left|\nu_{A}^{+}(x)-v_{B}^{+}(x)\right|\right]^{2}}{2}, \\
& v_{f(A, B)}^{-}(x) \\
& \quad=\frac{1-\left[\left|\mu_{A}^{-}(x)-\mu_{B}^{-}(x)\right| \vee\left|\nu_{A}^{+}(x)-v_{B}^{+}(x)\right|\right]^{2}}{2}, \\
& v_{f(A, B)}^{+}(x) \\
& \quad=\frac{1-\left[\left|\mu_{A}^{-}(x)-\mu_{B}^{-}(x)\right| \vee\left|\nu_{A}^{+}(x)-v_{B}^{+}(x)\right|\right]}{2} .
\end{aligned}
$$


Theorem 23 (see [36]). Let $E$ be an entropy on $\operatorname{IvIFS}(X)$. Then $E_{f}(A, B)=E(f(A, B)): \operatorname{IvIFS}(X) \times \operatorname{IvIFS}(X) \rightarrow[0,1]$ is a similarity measure on $\operatorname{IvIFS}(X)$.

Based on the compositive entropy measure of formula (17), we have following similarity measure of $A, B$ on $\operatorname{IvIFS}(X)$ :

$$
\begin{gathered}
E_{f}(A, B)=E(f(A, B))=\frac{2}{5 n \ln 2} \sum_{i=1}^{n}\left(\left(M_{i}^{2}-\frac{M_{i}}{4}\right)\right. \\
\cdot \ln 2+\left(\frac{M_{i}}{4}+\frac{M_{i}^{2}}{2}+\frac{M_{i}^{3}}{4}\right) \\
\cdot \ln \left(\frac{M_{i}}{4}+\frac{M_{i}^{2}}{2}+\frac{M_{i}^{3}}{4}\right) \\
+\left(\frac{M_{i}}{4}+\frac{M_{i}^{2}}{2}+\frac{M_{i}^{3}}{4}+1\right) \\
\left.\cdot \ln \frac{2}{M_{i} / 4+M_{i}^{2} / 2+M_{i}^{3} / 4+1}\right)
\end{gathered}
$$

where $M_{i}=\left|\mu_{A}^{-}\left(x_{i}\right)-\mu_{B}^{-}\left(x_{i}\right)\right| \vee\left|\nu_{A}^{+}\left(x_{i}\right)-\nu_{B}^{+}\left(x_{i}\right)\right|, i=1,2, \ldots, n$.

Example 24. Let us consider the following pattern recognition problem as discussed in [37]. Assume $R_{1}, R_{2}, R_{3}, R_{4}$, and $R_{5}$ are given five known patterns, which correspond to five decision alternatives $d_{1}, d_{2}, d_{3}, d_{4}$, and $d_{5}$, respectively. The patterns are denoted by the following IvIFSs in $X=$ $\left\{x_{1}, x_{2}, x_{3}, x_{4}\right\}$ :

$$
\begin{aligned}
R_{1} & =\left\{\left(x_{1},[0.4,0.5],[0.3,0.4]\right)\right. \\
& \left(x_{2},[0.4,0.6],[0.2,0.4]\right),\left(x_{3},[0.3,0.4],[0.4,0.5]\right) \\
& \left.\left(x_{4},[0.5,0.6],[0.1,0.3]\right)\right\} \\
R_{2} & =\left\{\left(x_{1},[0.5,0.6],[0.2,0.3]\right)\right. \\
& \left(x_{2},[0.6,0.7],[0.2,0.3]\right),\left(x_{3},[0.5,0.6],[0.3,0.4]\right) \\
& \left.\left(x_{4},[0.4,0.7],[0.1,0.2]\right)\right\} \\
R_{3} & =\left\{\left(x_{1},[0.3,0.5],[0.3,0.4]\right)\right. \\
& \left(x_{2},[0.1,0.3],[0.5,0.6]\right),\left(x_{3},[0.2,0.5],[0.4,0.5]\right) \\
& \left.\left(x_{4},[0.2,0.3],[0.4,0.6]\right)\right\} \\
R_{4} & =\left\{\left(x_{1},[0.2,0.5],[0.3,0.4]\right)\right. \\
& \left(x_{2},[0.4,0.7],[0.1,0.2]\right),\left(x_{3},[0.4,0.5],[0.3,0.5]\right) \\
& \left.\left(x_{4},[0.5,0.8],[0.1,0.2]\right)\right\} \\
R_{5} & =\left\{\left(x_{1},[0.3,0.4],[0.1,0.3]\right)\right. \\
& \left(x_{2},[0.7,0.8],[0.1,0.2]\right),\left(x_{3},[0.5,0.6],[0.2,0.4]\right) \\
& \left.\left(x_{4},[0.6,0.7],[0.1,0.2]\right)\right\}
\end{aligned}
$$

Given an unknown sample,

$$
\begin{aligned}
r^{+} & =\left\{\left(x_{1},[0.5,0.6],[0.1,0.3]\right)\right. \\
& \left(x_{2},[0.7,0.8],[0.1,0.2]\right),\left(x_{3},[0.5,0.6],[0.2,0.4]\right), \\
& \left.\left(x_{4},[0.6,0.7],[0.1,0.2]\right)\right\} .
\end{aligned}
$$

From formula (28), we can compute the degree of similarity between $R_{i}$ and $r^{+}$as follows:

$$
\begin{aligned}
& E_{f}\left(R_{1}, r^{+}\right)=0.3753, \\
& E_{f}\left(R_{2}, r^{+}\right)=0.3866, \\
& E_{f}\left(R_{3}, r^{+}\right)=0.2395, \\
& E_{f}\left(R_{4}, r^{+}\right)=0.2944, \\
& E_{f}\left(R_{5}, r^{+}\right)=0.4344 .
\end{aligned}
$$

Our aim is to classify pattern $r^{+}$to one of the decision alternatives $d_{1}, d_{2}, d_{3}, d_{4}$, and $d_{5}$. Since $E_{f}\left(R_{5}, r^{+}\right)=$ $\operatorname{Max}_{1 \leq i \leq 5} E_{f}\left(R_{i}, r^{+}\right)$, then the pattern $r^{+}$should be classified to $R_{5}$ according to the principle of maximum similarity. It means that the decision alternative $d_{5}$ is the optimal alternative which is the closest alterative to positive ideal solution. This result is in agreement with the one obtained in [37].

\section{Conclusions}

In this paper, we propose some kinds of entropy to measure the uncertain information of IvIFS. Firstly, we investigate the interval-valued intuitionistic fuzzy entropy by introducing the interval-valued intuitionistic fuzzy cross-entropy. Then we put forward three kinds of entropy of IvIFS, including interval-valued intuitionistic entropy, interval-valued fuzzy entropy, and interval-valued span entropy. Based on the three kinds of entropy, the compositive entropy of IvIFS is established. Furthermore, a measure formula of compositive entropy is proposed. At last, we compare the compositive entropy measure with other entropy measures and show the applications on multiple attributes' decision-making and pattern recognition. And the results are feasible.

\section{Conflict of Interests}

The authors declare that there is no conflict of interests regarding the publication of this paper.

\section{Acknowledgments}

The work is supported by the NNSF of China (no. 71371011), the Provincial Nature Science Research Key Project for Colleges and Universities of Anhui Province (no. KJ2013A033), the Talent Support Key Project for Outstanding Young of Colleges and Universities in 2016 (no. gxyqZD2016453), and 
the Project of Graduate Academic Innovation of Anhui University (no. yfc100023).

\section{References}

[1] L. A. Zadeh, "Fuzzy sets," Information and Control, vol. 8, pp. 338-353, 1965.

[2] K. T. Atanassov, "Intuitionistic fuzzy sets," Fuzzy Sets and Systems, vol. 20, no. 1, pp. 87-96, 1986.

[3] L. A. Zadeh, "The concept of a linguistic variable and its application to approximate reasoning I," Information Sciences, vol. 8, pp. 199-249, 1975.

[4] W.-L. Gau and D. J. Buehrer, "Vague sets," IEEE Transactions on Systems, Man and Cybernetics, vol. 23, no. 2, pp. 610-614, 1993.

[5] H. Bustince and P. Burillo, "Vague sets are intuitionistic fuzzy sets," Fuzzy Sets and Systems, vol. 79, no. 3, pp. 403-405, 1996.

[6] V. Torra, "Hesitant fuzzy sets," International Journal of Intelligent Systems, vol. 25, no. 6, pp. 529-539, 2010.

[7] K. T. Atanassov and G. Gargov, "Interval valued intuitionistic fuzzy sets," Fuzzy Sets and Systems, vol. 31, no. 3, pp. 343-349, 1989.

[8] K. T. Atanassov, "Operators over interval valued intuitionistic fuzzy sets," Fuzzy Sets and Systems, vol. 64, no. 2, pp. 159-174, 1994.

[9] D. G. Park, Y. C. Kwun, J. H. Park, and I. Y. Park, "Correlation coefficient of interval-valued intuitionistic fuzzy sets and its application to multiple attribute group decision making problems," Mathematical and Computer Modelling, vol. 50, no. 9-10, pp. 1279-1293, 2009.

[10] G. Deschrijver and E. E. Kerre, "On the position of intuitionistic fuzzy set theory in the framework of theories modelling imprecision," Information Sciences, vol. 177, no. 8, pp. 1860-1866, 2007.

[11] Z. S. Xu, J. Chen, and J. J. Wu, "Clustering algorithm for intuitionistic fuzzy sets," Information Sciences, vol. 178, no. 19, pp. 3775-3790, 2008.

[12] Z. S. Xu and J. Chen, "An overview of distance and similarity measures of intuitionistic fuzzy sets," International Journal of Uncertainty, Fuzziness and Knowledge-Based Systems, vol. 16, no. 4, pp. 529-555, 2008.

[13] L. A. Zadeh, "Probability measures of fuzzy events," Journal of Mathematical Analysis and Applications, vol. 23, pp. 421-427, 1968.

[14] A. de Luca and S. Termini, "A definition of a nonprobabilistic entropy in the setting of fuzzy sets theory," Information and Computation, vol. 20, pp. 301-312, 1972.

[15] A. Kaufmann, Introduction to the Theory of Fuzzy Sets: Fundamental Theoretical Elements, vol. 1, Academic Press, New York, NY, USA, 1975.

[16] R. R. Yager, "On the measure of fuzziness and negation. I. Membership in the unit interval," International Journal of General Systems, vol. 5, no. 4, pp. 221-229, 1979.

[17] X. C. Liu, "Entropy, distance measure and similarity measure of fuzzy sets and their relations," Fuzzy Sets and Systems, vol. 52, no. 3, pp. 305-318, 1992.

[18] P. Burillo and H. Bustince, "Estructuras algebraicas en conjuntos IFS," in I1 Conoreso Nacional de Lógica y Tecnologia Fuzzy, pp. 135-147, Boadilla del Monte, Madrid, Spain, 1992.
[19] P. Burillo and H. Bustince, "Entropy on intuitionistic fuzzy sets and on interval-valued fuzzy sets," Fuzzy Sets and Systems, vol. 78, no. 3, pp. 305-316, 1996.

[20] E. Szmidt and J. Kacprzyk, "Entropy for intuitionistic fuzzy sets," Fuzzy Sets and Systems, vol. 118, no. 3, pp. 467-477, 2001.

[21] W.-L. Hung and M.-S. Yang, "Fuzzy entropy on intuitionistic fuzzy sets," International Journal of Intelligent Systems, vol. 21, no. 4, pp. 443-451, 2006.

[22] Y. Wang and Y.-J. Lei, "A technique for constructing intuitionistic fuzzy entropy," Control and Decision, vol. 22, no. 12, pp. 13901394, 2007.

[23] J. Mao, D. Yao, and C. Wang, "A novel cross-entropy and entropy measures of IFSs and their applications," KnowledgeBased Systems, vol. 48, pp. 37-45, 2013.

[24] Q. Zhang, S. Jiang, B. Jia, and S. Luo, "Some information measures for interval-valued intuitionistic fuzzy sets," Information Sciences, vol. 180, no. 24, pp. 5130-5145, 2010.

[25] C.-P. Wei, P. Wang, and Y.-Z. Zhang, "Entropy, similarity measure of interval-valued intuitionistic fuzzy sets and their applications," Information Sciences, vol. 181, no. 19, pp. 42734286, 2011.

[26] Y. Zhang, P. Ma, X. H. Su, and C. Zhang, "Entropy on interval-valued intuitionistic fuzzy sets and its application in multi-attribute decision making," in Proceedings of the 14th International Conference on Information Fusion (FUSION '11), pp. 1-7, IEEE, Chicago, Ill, USA, July 2011.

[27] F. Jin, L. Pei, H. Chen, and L. Zhou, "Interval-valued intuitionistic fuzzy continuous weighted entropy and its application to multi-criteria fuzzy group decision making," Knowledge-Based Systems, vol. 59, pp. 132-141, 2014.

[28] J. Ye, "Multicriteria fuzzy decision-making method using entropy weights-based correlation coefficients of intervalvalued intuitionistic fuzzy sets," Applied Mathematical Modelling, vol. 34, no. 12, pp. 3864-3870, 2010.

[29] K. Qu, L. Tang, and W.-J. Rong, "The construction and basic properties of entropy of interval-valued intuitionistic fuzzy sets," Journal of Chongqing College of Liberal Arts: Natural Science Edition, vol. 3, pp. 21-24, 2010 (Chinese).

[30] X.-G. Shang and W.-S. Jiang, "A note on fuzzy information measures," Pattern Recognition Letters, vol. 18, no. 5, pp. 425432, 1997.

[31] I. K. Vlachos and G. D. Sergiadis, "Intuitionistic fuzzy information-applications to pattern recognition," Pattern Recognition Letters, vol. 28, no. 2, pp. 197-206, 2007.

[32] J.-J. Peng, J.-Q. Wang, X.-H. Wu, H.-Y. Zhang, and X.-H. Chen, "The fuzzy cross-entropy for intuitionistic hesitant fuzzy sets and their application in multi-criteria decision-making," International Journal of Systems Science, vol. 46, no. 13, pp. 23352350, 2015.

[33] J. Ye, "Fuzzy cross entropy of interval-valued intuitionistic fuzzy sets and its optimal decision-making method based on the weights of alternatives," Expert Systems with Applications, vol. 38, no. 5, pp. 6179-6183, 2011.

[34] J. Ye, "Fuzzy decision-making method based on the weighted correlation coefficient under intuitionistic fuzzy environment," European Journal of Operational Research, vol. 205, no. 1, pp. 202-204, 2010.

[35] G.-W. Wei, "Gray relational analysis method for intuitionistic fuzzy multiple attribute decision making," Expert Systems with Applications, vol. 38, no. 9, pp. 11671-11677, 2011. 
[36] K. Hu and J. Li, "The entropy and similarity measure of interval valued intuitionistic fuzzy sets and their relationship," International Journal of Fuzzy Systems, vol. 15, no. 3, pp. 279288, 2013.

[37] G. Wei and G. Lan, "Grey relational analysis method for interval-valued intuitionistic fuzzy multiple attribute decision making," in Proceedings of the 5th International Conference on Fuzzy Systems and Knowledge Discovery (FSKD '08), vol. 1, pp. 291-295, IEEE, Shandong, China, October 2008. 


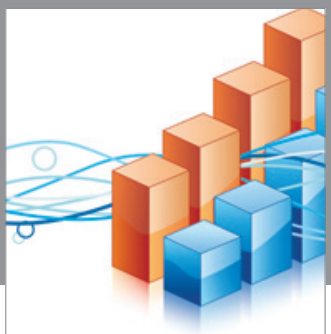

Advances in

Operations Research

vatem alat4

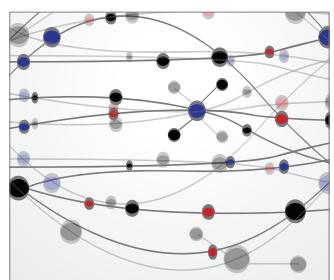

\section{The Scientific} World Journal
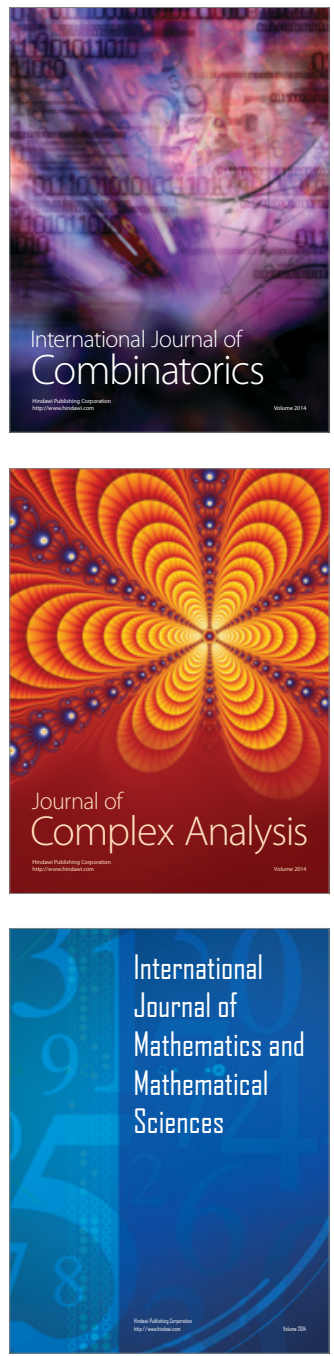
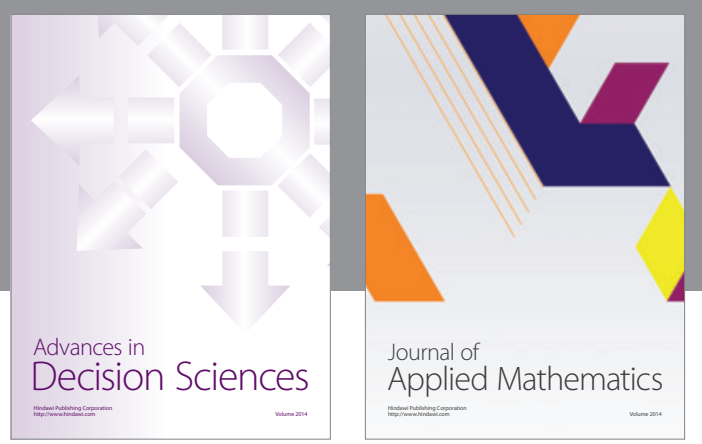

Algebra

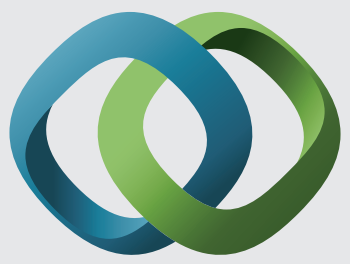

\section{Hindawi}

Submit your manuscripts at

http://www.hindawi.com
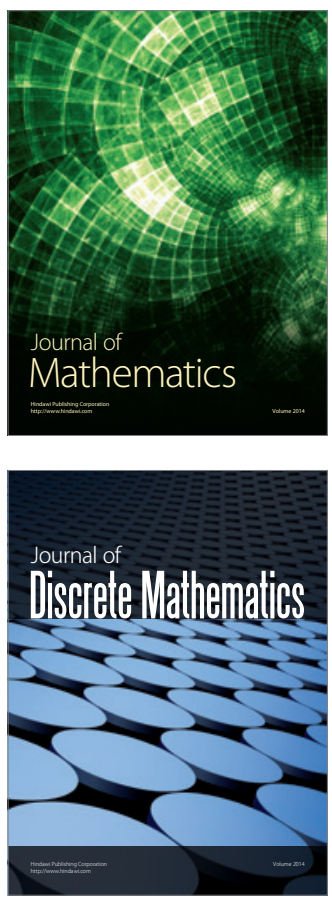

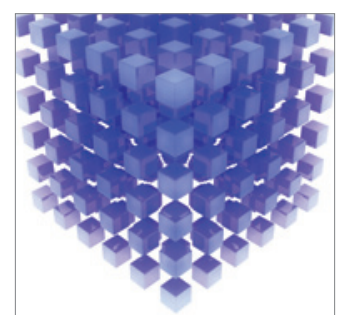

Mathematical Problems in Engineering
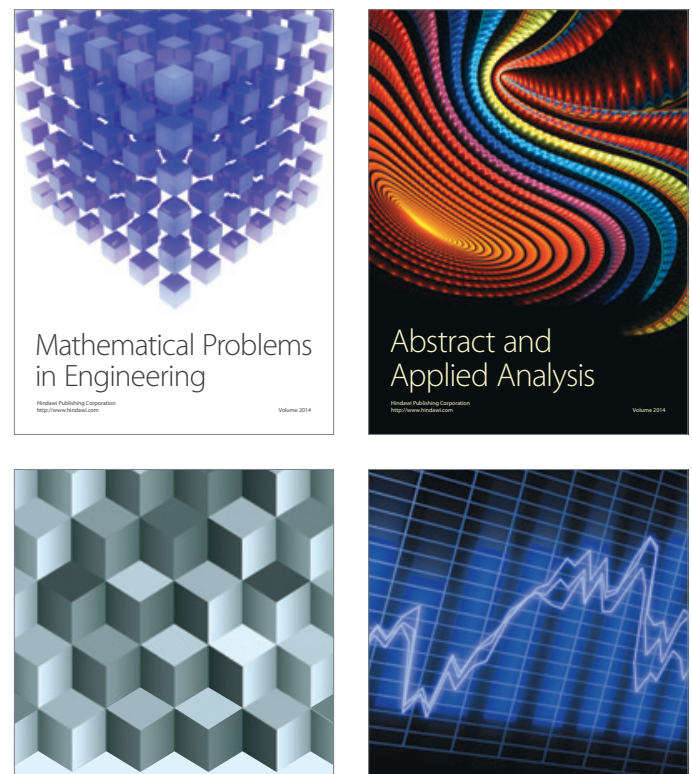

Journal of

Function Spaces

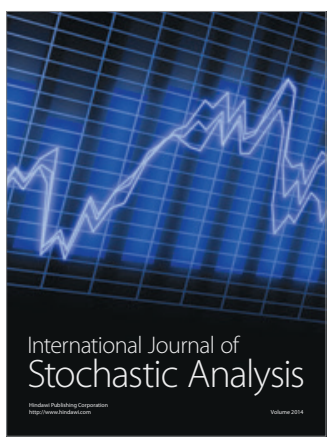

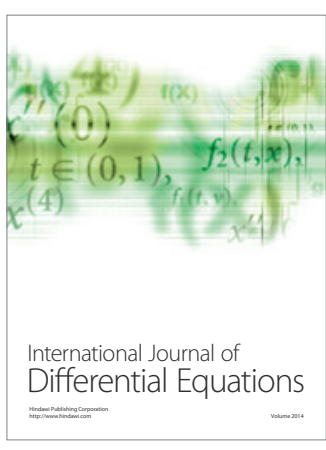
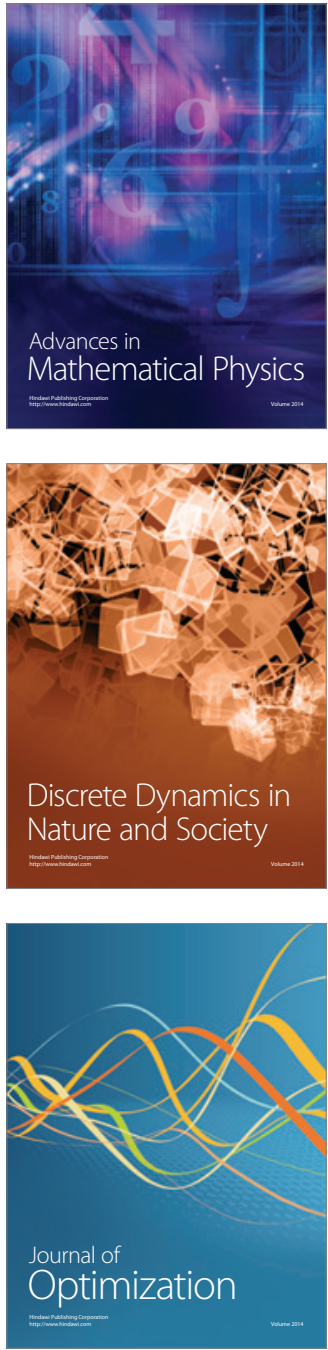